\title{
On-demand, cell-free biomanufacturing of conjugate vaccines at the point-of-care
}

Jessica C. Stark, ${ }^{1,2,3,}$ † Thapakorn Jaroentomeechai, ${ }^{4,}$ † Tyler D. Moeller, ${ }^{4}$ Rachel S. Dubner, ${ }^{5}$ Karen J. Hsu, ${ }^{6}$ Taylor C. Stevenson, ${ }^{7}$ Matthew P. DeLisa, ${ }^{4,7,{ }^{*}}$ and Michael C. Jewett ${ }^{1,2,3,8,9,10,{ }^{*}}$

${ }^{1}$ Department of Chemical and Biological Engineering, Northwestern University, 2145 Sheridan Rd Technological Institute E136, Evanston, IL USA, 60208-3120

${ }^{2}$ Center for Synthetic Biology, Northwestern University, 2145 Sheridan Rd Technological Institute E136, Evanston, IL USA, 60208-3120

${ }^{3}$ Chemistry of Life Processes Institute, Northwestern University, 2170 Campus Drive, Evanston, IL USA, 60208-3120

${ }^{4}$ Robert Frederick Smith School of Chemical and Biomolecular Engineering, Cornell University, 120 Olin Hall, Ithaca, NY USA, 14853

${ }^{5}$ Department of Biological Sciences, Northwestern University, 2205 Tech Drive Hogan Hall 2144, Evanston, IL USA, 60208-3500

${ }^{6}$ Department of Mechanical Engineering, Northwestern University, 2145 Sheridan Rd Technological Institute B224, Evanston, IL USA, 60208-3120

${ }^{7}$ Nancy E. and Peter C. Meinig School of Biomedical Engineering, Cornell University, Weill Hall, Ithaca, NY USA, 14853

${ }^{8}$ Robert H. Lurie Comprehensive Cancer Center, Northwestern University, 676 N. St Clair St, Suite 1200, Chicago, IL, USA, 60611-3068

${ }^{9}$ Simpson-Querrey Institute, Northwestern University, 303 E. Superior St, Suite 11-131 Chicago, IL USA, 60611-2875

${ }^{10}$ Lead contact

$\dagger$ These authors contributed equally to this work

*Correspondence to:

Dr. Matthew P. DeLisa

Robert Frederick Smith School of Chemical and Biomolecular Engineering

Cornell University

254 Olin Hall

Ithaca, NY 14853

Tel: +1 607-254-8560

Email: md255@cornell.edu

Dr. Michael C. Jewett

Department of Chemical and Biological Engineering

Northwestern University

2145 Sheridan Road, Technological Institute E136

Evanston, IL 60208-3120

Tel: +1 847-467-5007

E-mail: m-jewett@northwestern.edu 


\section{Summary}

Conjugate vaccines are among the most effective methods for preventing bacterial infections, representing a promising strategy to combat drug-resistant pathogens. However, existing manufacturing approaches limit access to conjugate vaccines due to centralized production and cold chain distribution requirements. To address these limitations, we developed a modular technology for in vitro bioconjugate vaccine expression (iVAX) in portable, freeze-dried lysates from detoxified, nonpathogenic Escherichia coli. Upon rehydration, iVAX reactions synthesize clinically relevant doses of bioconjugate vaccines against diverse bacterial pathogens in one hour. We show that iVAX synthesized vaccines against the highly virulent pathogen Franciscella tularensis subsp. tularensis (type A) strain Schu S4 elicited pathogen-specific antibodies in mice at significantly higher levels compared to vaccines produced using engineered bacteria. The iVAX platform promises to accelerate development of new bioconjugate vaccines with increased access through refrigeration-independent distribution and point-of-care production.

\section{Keywords}

Conjugate vaccines, cell-free protein synthesis, synthetic biology, on-demand biosynthesis, decentralized biomanufacturing, cell-free glycoprotein synthesis, freeze-dried, Franciscella tularensis Schu S4, Escherichia coli O78, Escherichia coli O7 


\section{Introduction}

Drug-resistant bacteria are predicted to threaten up to 10 million lives per year by 2050 (The Review on Antimicrobial Resistance, 2014), necessitating new strategies to develop and distribute antibiotics and vaccines. Conjugate vaccines, typically composed of a pathogen-specific capsular (CPS) or O-antigen polysaccharide (O-PS) linked to an immunostimulatory protein carrier, are among the safest and most effective methods for preventing life-threatening bacterial infections (Jin et al., 2017; Trotter et al., 2008; Weintraub, 2003). In particular, implementation of meningococcal and pneumococcal conjugate vaccines have significantly reduced the occurrence of bacterial meningitis and pneumonia worldwide (Novak et al., 2012; Poehling et al., 2006), in addition to reducing antibiotic resistance in targeted strains (Roush et al., 2008). However, despite their proven safety and efficacy, global childhood vaccination rates for conjugate vaccines remain as low as $\sim 30 \%$, with lack of access or low immunization coverage accounting for the vast majority of remaining disease burden (Wahl et al., 2018). In addition, the 2018 WHO prequalification of Typhbar-TCV ${ }^{\circledR}$ to prevent typhoid fever represents the first conjugate vaccine approval in nearly a decade. In order to address emerging drug-resistant pathogens, new technologies to accelerate the development and global distribution of conjugate vaccines are needed.

Contributing to the slow pace of conjugate vaccine development and distribution is the fact that these molecules are particularly challenging and costly to manufacture. The conventional process to produce conjugate vaccines involves chemical conjugation of carrier proteins with polysaccharide antigens purified from large-scale cultures of pathogenic bacteria. Large-scale fermentation of pathogens results in high manufacturing costs due to associated biosafety hazards and process development challenges. In addition, chemical conjugation can alter the structure of the polysaccharide, resulting in loss of the protective epitope (Bhushan et al., 1998). To address these challenges, it was recently demonstrated that polysaccharide-protein "bioconjugates" can be made in Escherichia coli using protein-glycan coupling technology (PGCT) 
(Feldman et al., 2005). In this approach, engineered E. coli cells covalently attach heterologously expressed CPS or O-PS antigens to carrier proteins via an asparagine-linked glycosylation reaction catalyzed by the Campylobacter jejuni oligosaccharyltransferase enzyme PglB (CjPglB) (Cuccui et al., 2013; Garcia-Quintanilla et al., 2014; Ihssen et al., 2010; Ma et al., 2014; Marshall et al., 2018; Wacker et al., 2014; Wetter et al., 2013). Despite this advance, both chemical conjugation and PGCT approaches rely on living bacterial cells, requiring centralized production facilities from which vaccines are distributed via a refrigerated supply chain. Refrigeration is critical to avoid conjugate vaccine spoilage due to precipitation and significant loss of the pathogen-specific polysaccharide upon both heating and freezing (Frasch, 2009; WHO, 2014). Only one conjugate vaccine, MenAfriVac ${ }^{\mathrm{TM}}$, is known to remain active outside of the cold chain for up to 4 days, which enabled increased vaccine coverage and an estimated $50 \%$ reduction in costs during vaccination in the meningitis belt of sub-Saharan Africa (Lydon et al., 2014). However, this required significant investment in the development and validation of a thermostable vaccine. Broadly, the need for cold chain refrigeration creates economic and logistical challenges that limit the reach of vaccination campaigns and present barriers to the eradication of disease, especially in the developing world (Ashok et al., 2017; Wahl et al., 2018).

Cell-free protein synthesis (CFPS) offers opportunities to both accelerate vaccine development and enable decentralized, cold chain-independent biomanufacturing by using cell Iysates, rather than living cells, to synthesize proteins in vitro (Carlson et al., 2012). Importantly, CFPS platforms (i) enable point-of-care protein production, as relevant amounts of protein can be synthesized in vitro in just a few hours, (ii) can be freeze-dried for distribution at ambient temperature and reconstituted by just adding water (Adiga et al., 2018; Pardee et al., 2016), and (iii) circumvent biosafety concerns associated with the use of living cells outside of a controlled laboratory setting. CFPS has recently been used to enable on-demand and portable production of aglycosylated protein vaccines (Adiga et al., 2018; Pardee et al., 2016). Moreover, we recently described a cell-free glycoprotein synthesis technology that enables one-pot production of 
glycosylated proteins, including human glycoproteins and eukaryotic glycans (Jaroentomeechai et al., 2018). Despite these advances, cell-free systems and even decentralized manufacturing systems have historically been limited by their inability to synthesize glycosylated proteins at relevant titers and bearing diverse glycan structures, such as the polysaccharide antigens needed for bioconjugate vaccine production (Perez et al., 2016).

To address these limitations, here we describe the iVAX (in vitro bioconjugate vaccine expression) platform that enables rapid development and cold chain-independent biosynthesis of conjugate vaccines in cell-free reactions (Figure 1). iVAX was designed to have the following features. First, iVAX is fast, with the ability to produce multiple individual doses of bioconjugates in one hour. Second, iVAX is robust, yielding equivalent amounts of bioconjugate over a range of operating temperatures. Third, iVAX is modular, offering the ability to rapidly interchange carrier proteins, including those used in licensed conjugate vaccines, as well as conjugated polysaccharide antigens. We leverage this modularity to create an array of vaccine candidates targeted against diverse bacterial pathogens, including the highly virulent Franciscella tularensis subsp. tularensis (type A) strain Schu S4, enterotoxigenic (ETEC) E. coli O78, and uropathogenic (UPEC) E. coli O7. Fourth, iVAX is shelf-stable, derived from freeze-dried cell-free reactions that operate in a just-add-water strategy. Fifth, iVAX is safe, leveraging lipid A remodeling that effectively avoids the high levels of endotoxin present in non-engineered $E$. coli manufacturing platforms. Our results demonstrate that anti-F. tularensis bioconjugates derived from freeze-dried, low-endotoxin iVAX reactions elicit pathogen-specific antibody responses in mice and outperform a bioconjugate produced using the established PGCT approach in living cells. Overall, the iVAX platform offers a new way to deliver the protective benefits of an important class of antibacterial vaccines to both the developed and developing world. 


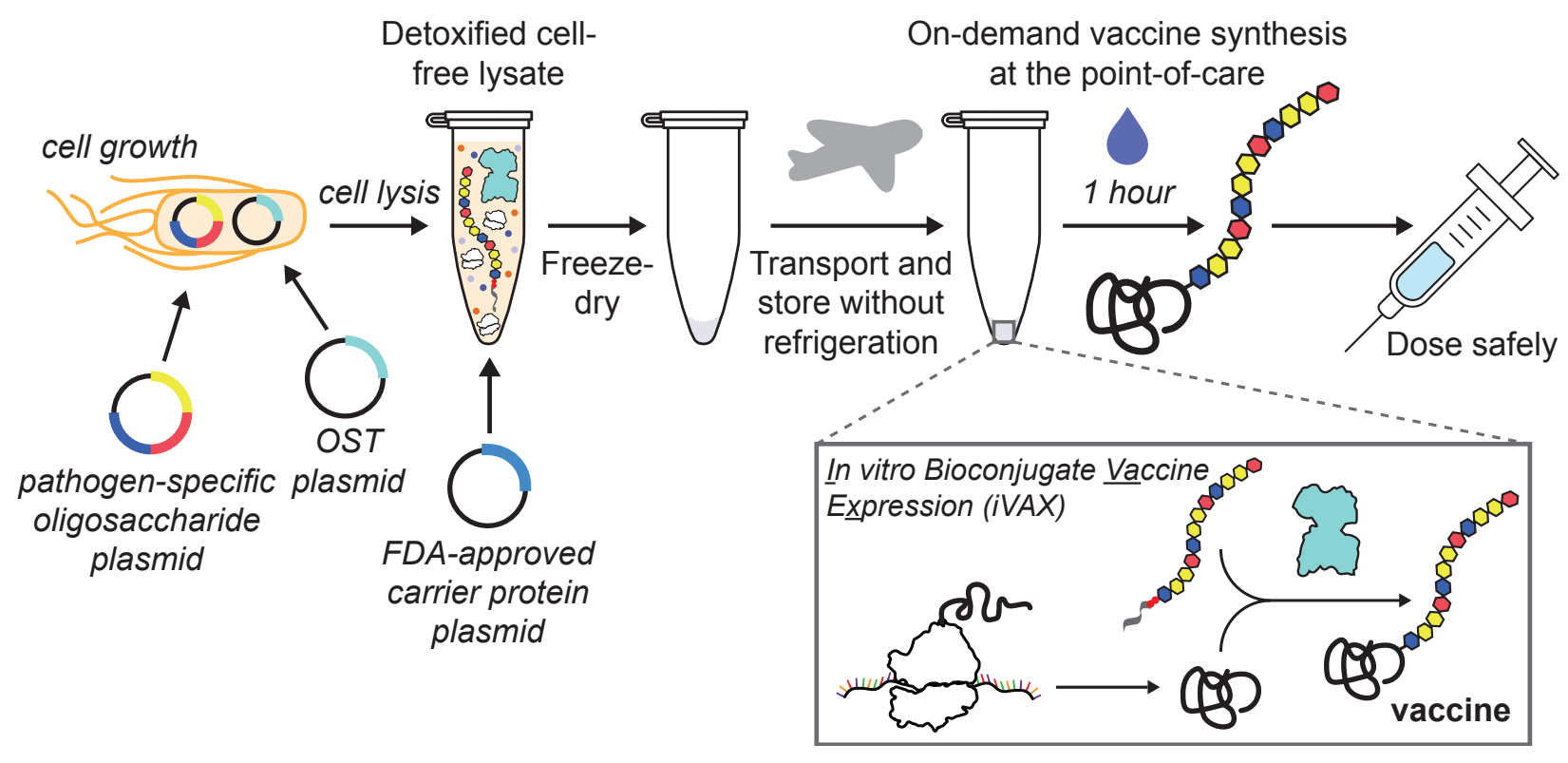

Figure 1. iVAX platform enables on-demand and portable production of antibacterial vaccines. The in vitro bioconjugate vaccine expression (iVAX) platform provides a rapid means to develop and distribute vaccines against bacterial pathogens. Expression of pathogen-specific polysaccharides (e.g., CPS, O-PS) and a bacterial oligosaccharyltransferase enzyme in engineered nonpathogenic $E$. coli with detoxified lipid A yields low-endotoxin lysates containing all of the machinery required for synthesis of bioconjugate vaccines. Reactions catalyzed by iVAX lysates can be used to produce bioconjugates containing licensed carrier proteins and can be freeze-dried without loss of activity for refrigeration-free transportation and storage. Freeze-dried reactions can be activated at the point-of-care via simple rehydration and used to reproducibly synthesize immunologically active bioconjugates in $\sim 1 \mathrm{~h}$.

\section{Results}

In vitro synthesis of licensed vaccine carrier proteins. To demonstrate proof-of-principle for cell-free bioconjugate vaccine production, we first set out to express a set of carrier proteins that are currently used in FDA-approved conjugate vaccines. Producing these carrier proteins in soluble conformations in vitro represented an important benchmark because their expression in living $E$. coli has proven challenging, often requiring purification and refolding of insoluble product from inclusion bodies (Haghi et al., 2011; Stefan et al., 2011), fusion of expression partners such as maltose-binding protein (MBP) to increase soluble expression (Figueiredo et al., 1995; Stefan et al., 2011), or expression of truncated protein variants in favor of the full-length proteins (Figueiredo et al., 1995). In contrast, cell-free protein synthesis approaches have recently shown 
promise for difficult-to-express proteins (Perez et al., 2016). The carrier proteins that we focused on here included nonacylated $H$. influenzae protein $D(P D)$, the $N$. meningitidis porin protein (PorA), and genetically detoxified variants of the Corynebacterium diphtheriae toxin (CRM197) and the Clostridium tetani toxin (TT). We also tested expression of the fragment C (TTC) and light chain (TTlight) domains of TT as well as E. coli MBP. While MBP is not a licensed carrier, it has demonstrated immunostimulatory properties (Fernandez et al., 2007) and when linked to O-PS was found to elicit polysaccharide-specific humoral and cellular immune responses in mice (Ma et al., 2014). Similarly, the TT domains, TTlight and TTc, have not been used in licensed vaccines, but are immunostimulatory and individually sufficient for protection against $C$. tetani challenge in mice (Figueiredo et al., 1995). To enable glycosylation, all carriers were modified at their C-termini with 4 tandem repeats of an optimal bacterial glycosylation motif, DQNAT (Chen et al., 2007). A C-terminal 6xHis tag was also included to enable purification and detection via Western blot analysis. A variant of superfolder green fluorescent protein that contained an internal DQNAT glycosylation site (sfGFP ${ }^{217-D Q N A T}$ ) (Jaroentomeechai et al., 2018) was used as a model protein to facilitate system development.

All eight carriers were synthesized in vitro with soluble yields of $\sim 50-650 \mu \mathrm{gL} \mathrm{m}^{-1}$ as determined by ${ }^{14} \mathrm{C}$-leucine incorporation (Figure 2a). In particular, the MBP ${ }^{4 \times D Q N A T}$ and $P D^{4 \times D Q N A T}$ variants were nearly $100 \%$ soluble, with yields of $500 \mu \mathrm{g} \mathrm{mL}^{-1}$ and $200 \mu \mathrm{g} \mathrm{mL}^{-1}$, respectively, and expressed as exclusively full-length products according to Western blot analysis (Figure 2b). Notably, similar soluble yields were observed for all carriers at $25^{\circ} \mathrm{C}, 30^{\circ} \mathrm{C}$, and $37^{\circ} \mathrm{C}$, with the exception of CRM197 ${ }^{4 \times D Q N A T}$ (Figure S1a), which is known to be heat sensitive (WHO, 2014). These results suggest that our method of cell-free carrier biosynthesis is robust over a $13^{\circ} \mathrm{C}$ range in temperature and could be used in settings where precise temperature control is not feasible.

The open reaction environment of our cell-free reactions enabled facile manipulation of the chemical and reaction environment to improve production of more complex carriers. For example, in the case of the membrane protein PorA ${ }^{4 \times D Q N A T}$, lipid nanodiscs were added to 
increase soluble expression (Figure S1b). Nanodiscs provide a cellular membrane mimic to cotranslationally stabilize hydrophobic regions of membrane proteins (Bayburt and Sligar, 2010). For expression of TT, which contains an intermolecular disulfide bond, expression was carried out for 2 hours in oxidizing conditions (Knapp et al., 2007), which improved assembly of the heavy and light chains into full-length product and minimized protease degradation of full-length TT (Figure S1c). In vitro synthesized CRM197 ${ }^{4 \times D Q N A T}$ and $\mathrm{TT}^{4 \times D Q N A T}$ were comparable in size to commercially available purified diphtheria toxin (DT) and TT protein standards and were reactive with $\alpha$-DT and $\alpha$-TT antibodies, respectively (Figure S1d, e), indicating that both were produced in immunologically relevant conformations. This is notable as CRM197 and TT are FDA-approved vaccine antigens for diphtheria and tetanus, respectively, when they are administered without conjugated polysaccharides. Together, our results highlight the ability of CFPS to express licensed conjugate vaccine carrier proteins in soluble conformations over a range of temperatures. 


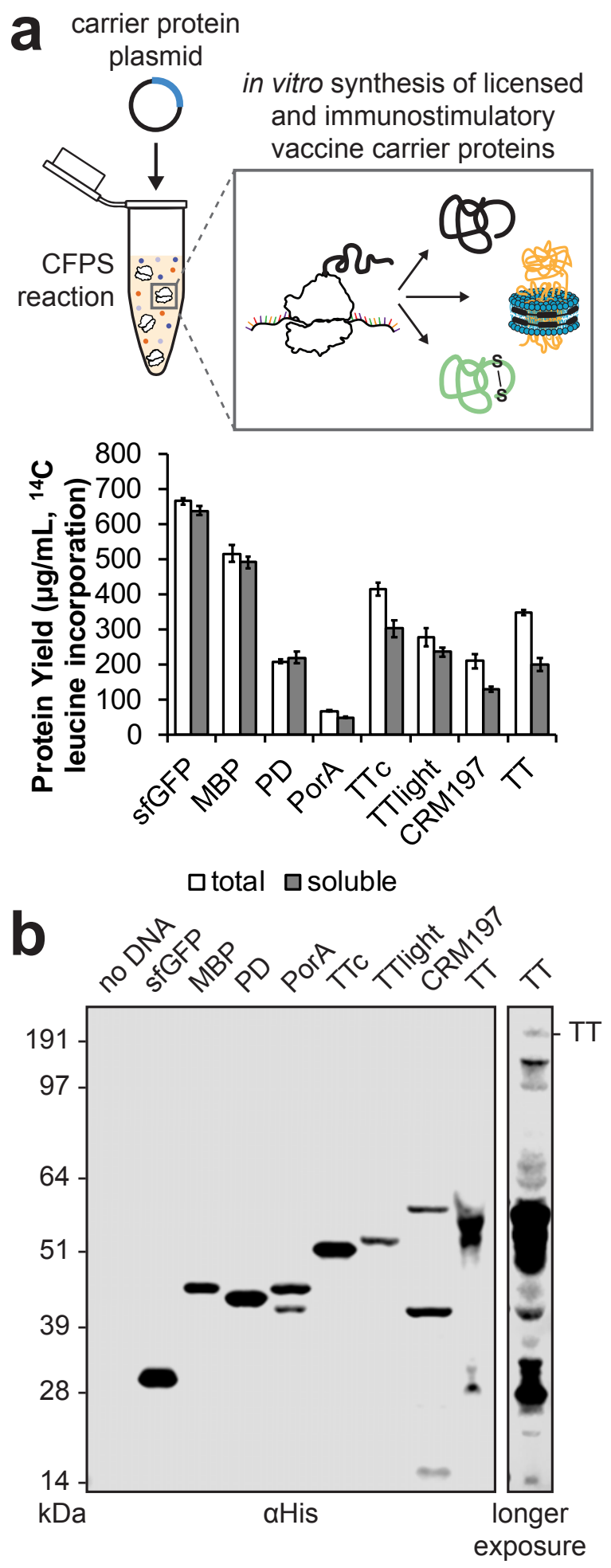

Figure 2. In vitro synthesis of licensed conjugate vaccine carrier proteins. (a) All four carrier proteins used in FDA-approved conjugate vaccines were synthesized solubly in vitro, as measured via ${ }^{14} \mathrm{C}$-leucine incorporation. These include $H$. influenzae protein $\mathrm{D}(\mathrm{PD})$, the $N$. meningitidis porin protein (PorA), and genetically detoxified variants of the $C$. diphtheriae toxin (CRM197) and the C. tetani toxin (TT). Additional immunostimulatory carriers were also 
synthesized solubly, including E. coli maltose binding protein (MBP) and the fragment C (TTC) and light chain (TTlight) domains of TT. Values represent means and error bars represent standard deviations of biological replicates $(n=3)$. (b) Full length product was observed for all proteins tested via Western blot. Different exposures are indicated with solid lines. Molecular weight ladder is shown at left. See also Figure S1.

On-demand synthesis of bioconjugate vaccines. We next sought to synthesize polysaccharide-conjugated versions of these carrier proteins by merging their in vitro expression with one-pot, cell-free glycosylation. As a model vaccine target, we focused on the highly virulent Francisella tularensis subsp. tularensis (type A) strain Schu S4, a gram-negative, facultative coccobacillus and the causative agent of tularemia. This bacterium is categorized as a class $\mathrm{A}$ bioterrorism agent due to its high fatality rate, low dose of infection, and ability to be aerosolized (Oyston et al., 2004). Although there are currently no licensed vaccines against $F$. tularensis, several studies have independently confirmed the important role of antibodies directed against $F$. tularensis LPS, specifically the O-PS repeat unit, in providing protection against the Schu S4 strain (Fulop et al., 2001; Lu et al., 2012). More recently, a bioconjugate vaccine comprising the F. tularensis Schu S4 O-PS (FtO-PS) conjugated to the Pseudomonas aeruginosa exotoxin A (EPA $^{\text {DNNNS-DQNRT }}$ ) carrier protein produced using PGCT (Cuccui et al., 2013; Marshall et al., 2018) was shown to be protective against challenge with the Schu S4 strain in a rat inhalation model of tularemia (Marshall et al., 2018). In light of these earlier findings, we investigated the ability of the iVAX platform to produce anti-F. tularensis bioconjugate vaccine candidates on-demand by conjugating the FtO-PS structure to diverse carrier proteins in vitro.

The FtO-PS is composed of the 826-Da repeating tetrasaccharide unit Qui4NFm(GalNAcAN)2-QuiNAc (Qui4NFm: 4,6-dideoxy-4-formamido-D-glucose; GalNAcAN: 2-acetamido2-deoxy-D-galacturonamide; QuiNAc: 2-acetamido-2,6-dideoxy-D-glucose) (Prior et al., 2003). To glycosylate proteins with FtO-PS, we produced an iVAX lysate from glycoengineered E. coli cells expressing the FtO-PS biosynthetic pathway and the oligosaccharyltransferase enzyme CjPgIB (Figure 3a). This lysate, which contained lipid-linked FtO-PS and active CjPgIB, was used 
to catalyze iVAX reactions primed with plasmid DNA encoding sfGFP $217-$ DQNAT . Control reactions in which attachment of the FtO-PS was not expected were performed with lysates from cells that lacked either the FtO-PS pathway or the CjPgIB enzyme. We also tested reactions that lacked plasmid encoding the target protein sfGFP $^{217-D Q N A T}$ or were primed with plasmid encoding sfGFP $217-A Q N A T$, which contained a mutated glycosylation site (AQNAT) that is not modified by CjPgIB (Kowarik et al., 2006). In reactions containing the iVAX lysate and primed with plasmid encoding SfGFP $217-$ DQNAT, immunoblotting with anti-His antibody or a commercial monoclonal antibody specific to FtO-PS revealed a ladder-like banding pattern (Figure $\mathbf{3 b}$ ). This ladder is characteristic of FtO-PS attachment, resulting from O-PS chain length variability through the action of the Wzy polymerase (Cuccui et al., 2013; Feldman et al., 2005; Prior et al., 2003). Glycosylation of sfGFP ${ }^{217-D Q N A T}$ was observed only in reactions containing a complete glycosylation pathway and the preferred DQNAT glycosylation sequence (Figure $\mathbf{3 b}$ ). This glycosylation profile was reproducible across biological replicates from the same lot of lysate (Figure 3c, left) and using different lots of lysate (Figure 3c, right). In vitro protein synthesis and glycosylation was observed after 1 hour, with the amount of conjugated polysaccharide reaching a maximum between 0.75 and 1.25 hours (Figure S2). Similar glycosylation reaction kinetics were observed at $37^{\circ} \mathrm{C}, 30^{\circ} \mathrm{C}, 25^{\circ} \mathrm{C}$, and room temperature $\left(\sim 21^{\circ} \mathrm{C}\right)$, indicating that iVAX reactions are robust over a range of temperatures (Figure S2). 


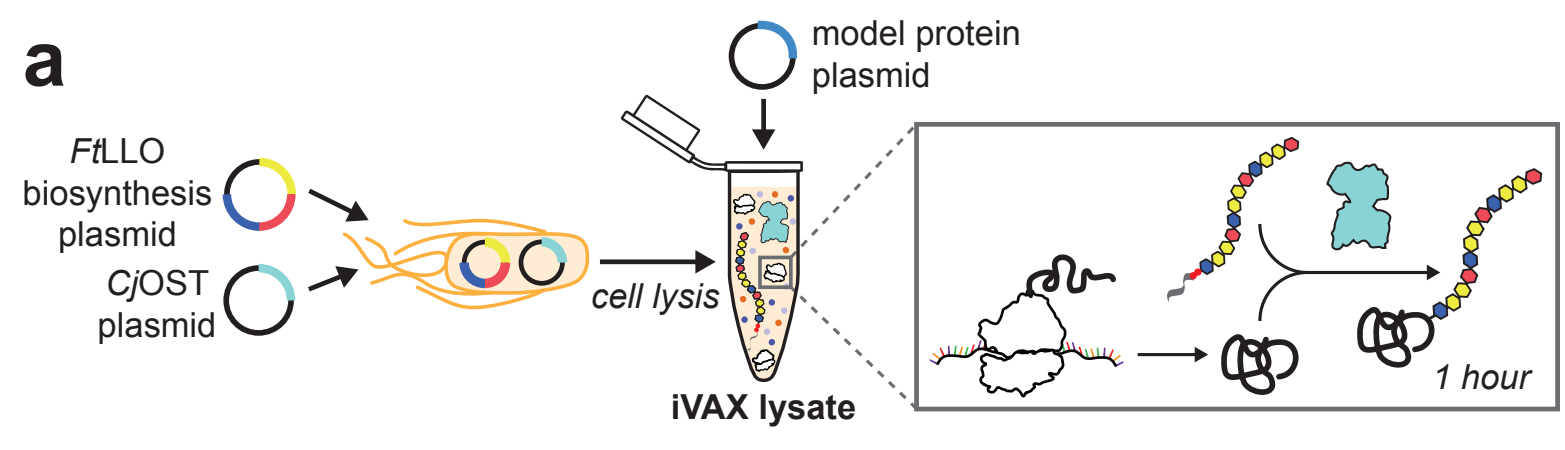

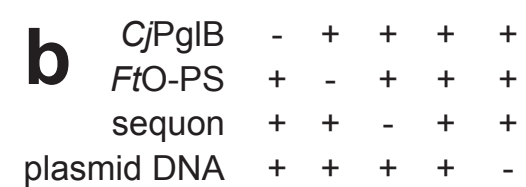
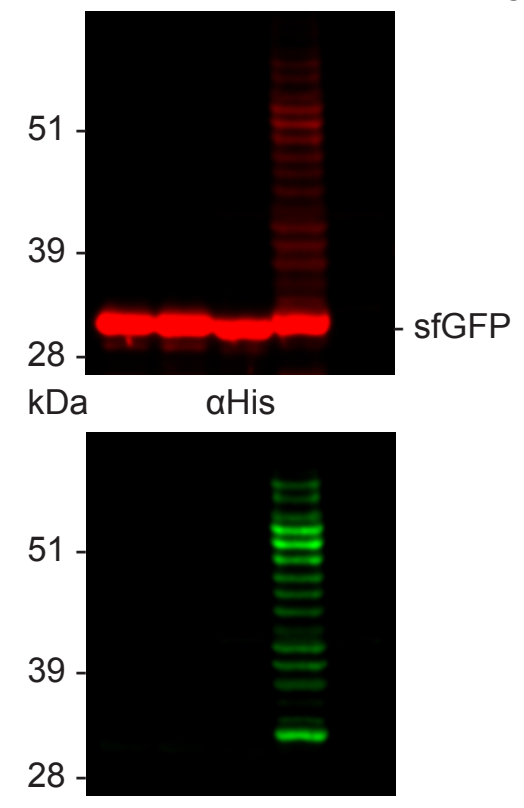

$\mathrm{kDa} \quad \alpha F t O-P S$

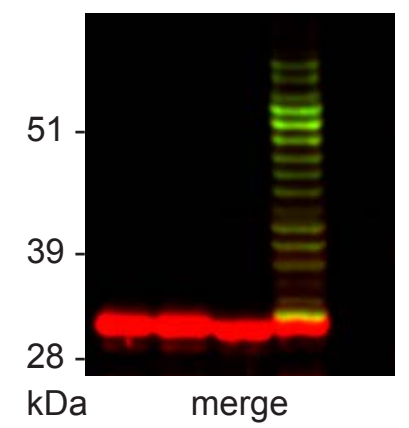

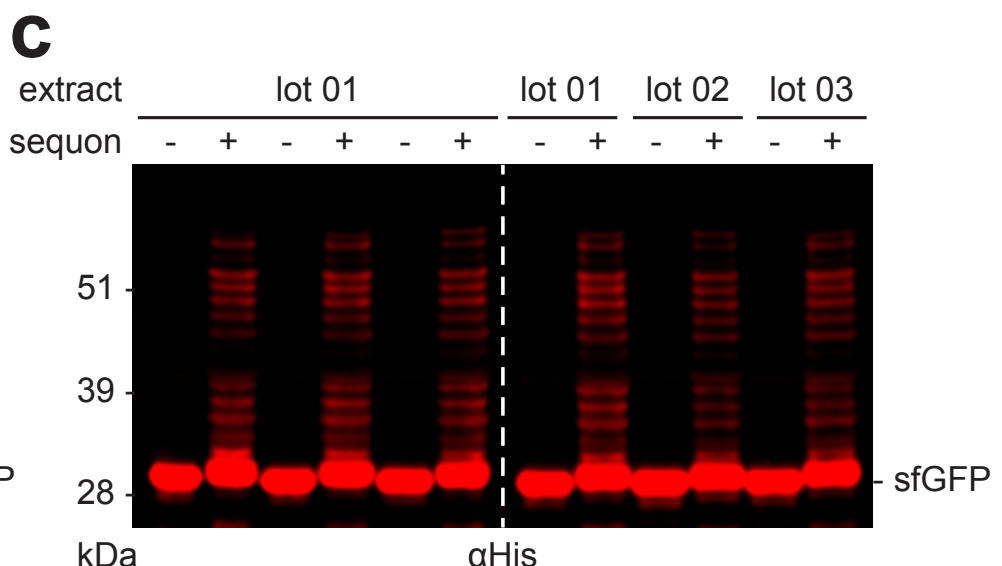

$\mathrm{kDa}$

aHis
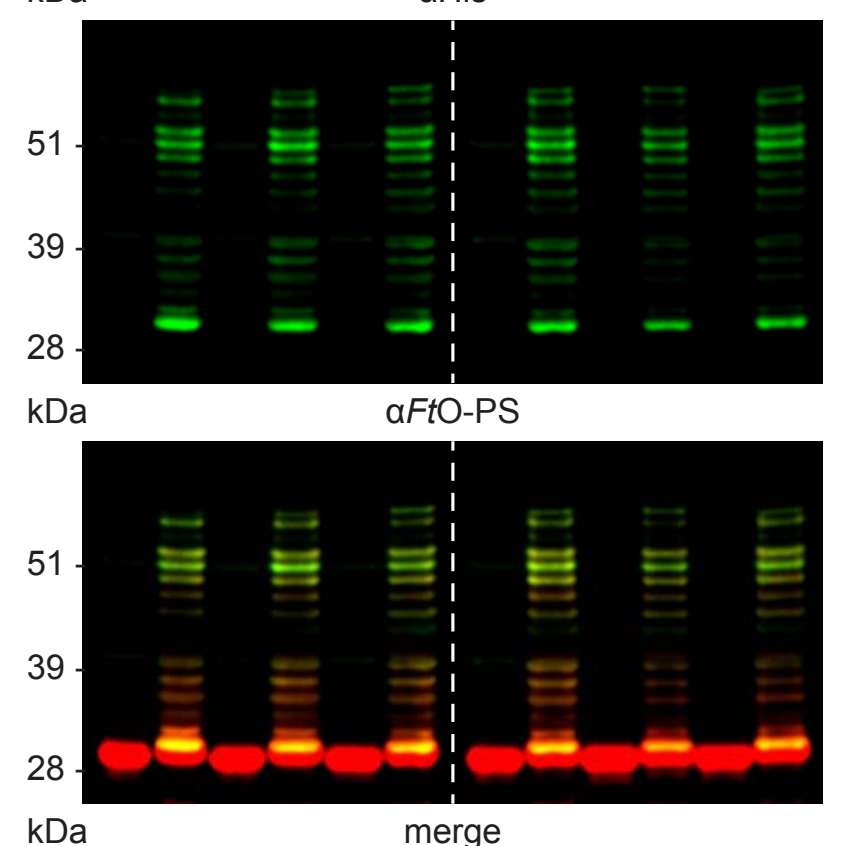

Figure 3. Reproducible glycosylation of proteins with FtO-PS in iVAX lysates. (a) iVAX lysates were prepared from cells expressing CjPgIB and a biosynthetic pathway encoding FtOPS. (b) Glycosylation of sfGFP ${ }^{217-D Q N A T}$ with FtO-PS was only observed when CjPgIB, FtO-PS, and the preferred sequon were present in the reaction (lane 3). When plasmid DNA was omitted, sfGFP ${ }^{217-D Q N A T}$ synthesis was not observed. (c) Biological replicates of iVAX reactions producing sfGFP $217-D Q N A T$ using the same lot (left) or different lots (right) of iVAX lysates demonstrated reproducibility of reactions and lysate preparation. Top panels show signal from probing with anti- 
hexa-histidine antibody (aHis) to detect the carrier protein, middle panels show signal from probing with commercial anti-FtO-PS antibody ( $\alpha$ FtO-PS), and bottom panels show aHis and $\alpha F t O-P S$ signals merged. Unless replicates are explicitly shown, images are representative of at least three biological replicates. Dashed lines indicate samples are from the same blot with the same exposure. Molecular weight ladders are shown at the left of each image. See also Figure S2.

Next, we investigated whether FDA-approved carriers could be similarly conjugated with FtO-PS in iVAX reactions. Following addition of plasmid DNA encoding MBP ${ }^{4 \times D Q N A T}, \mathrm{PD}^{4 \times D Q N A T}$, PorA ${ }^{4 \times D Q N A T}, T T c^{4 \times D Q N A T}$, TTlight ${ }^{4 \times D Q N A T}$, and CRM197 ${ }^{4 \times D Q N A T}$, glycosylation of each with FtO-PS was observed for iVAX reactions enriched with lipid-linked FtO-PS and CjPglB but not control reactions lacking CjPgIB (Figure 4). We observed conjugation of high molecular weight FtO-PS species (on the order of $\sim 10-20 \mathrm{kDa}$ ) to all protein carriers tested, which is important as longer glycan chain length has been shown to increase the efficacy of conjugate vaccines against $F$. tularensis (Stefanetti et al., 2019). Notably, our attempts to synthesize the same panel of bioconjugates using the established PGCT approach in living E. coli yielded less promising results. Specifically, we observed low levels of FtO-PS glycosylation and lower molecular weight conjugated FtO-PS species for all PGCT-derived bioconjugates compared to their iVAX-derived counterparts (Figure S3). The same trend was observed for PGCT- versus iVAX-derived bioconjugates involving the most common PGCT carrier protein, EPA ${ }^{\text {DNNNS-DQNRT }}$ (Cuccui et al., 2013; Ihssen et al., 2010; Marshall et al., 2018; Wacker et al., 2014; Wetter et al., 2013) (Figure 4; Figure S3). In addition, only limited expression of the PorA membrane protein was achieved in vivo (Figure S3). Collectively, these data indicate that iVAX could provide advantages over PGCT for production of bioconjugate vaccine candidates with high molecular weight O-PS antigens conjugated efficiently to diverse and potentially membrane-bound carrier proteins. 


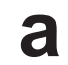

immunostimulatory carrier protein plasmid
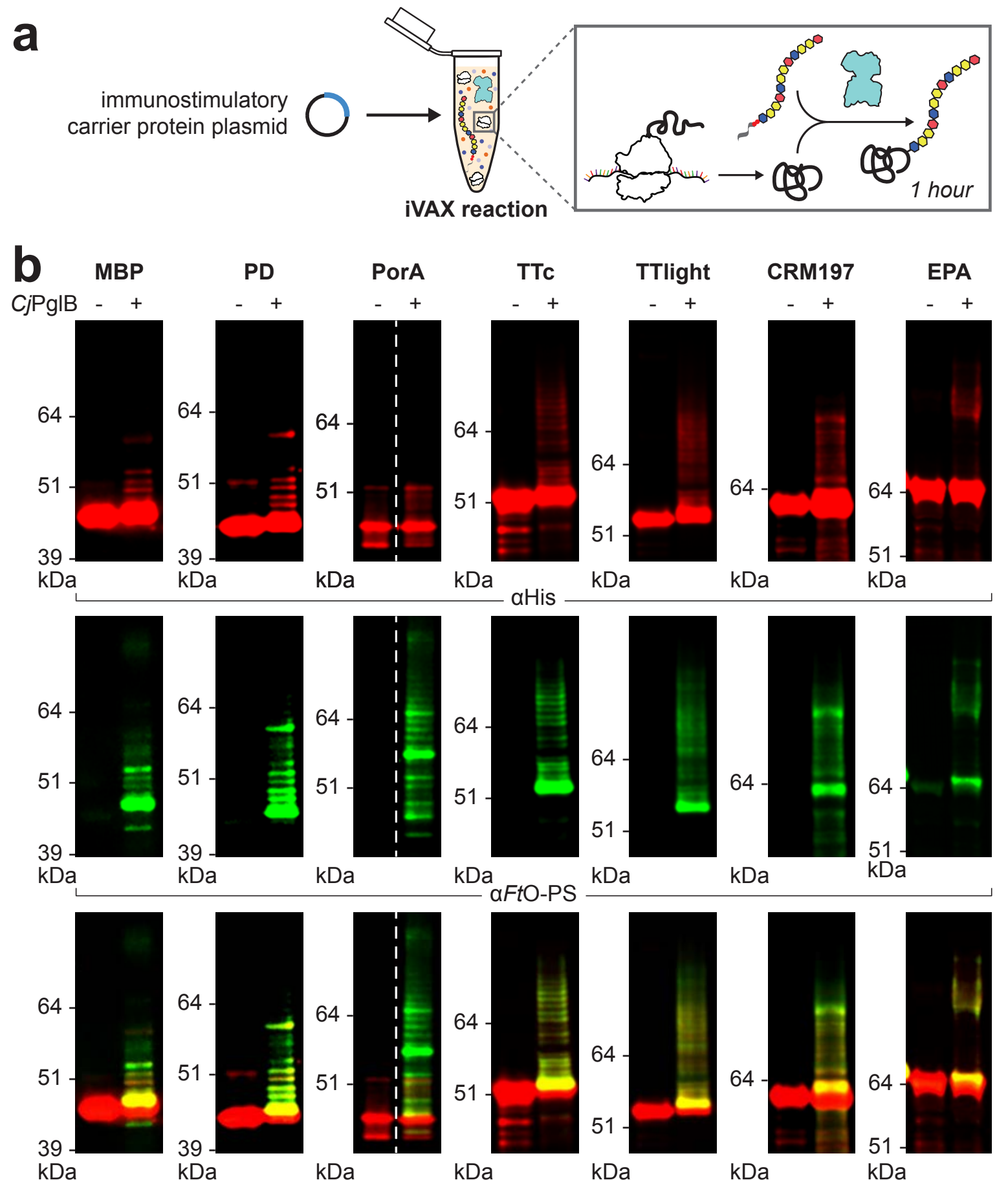

$\mathrm{kDa}$

$\mathrm{kDa}$

$\mathrm{kDa}$

$\mathrm{kDa}$

$\mathrm{kDa}$

$\mathrm{kDa}$

Figure 4. On-demand production of bioconjugates against $F$. tularensis using iVAX. (a) iVAX reactions were prepared from lysates containing CjPgIB and FtO-PS and primed with plasmid encoding immunostimulatory carriers, including those used in licensed vaccines. (b) We observed on-demand synthesis of anti- $F$. tularensis bioconjugate vaccines for all carrier proteins tested. Bioconjugates were purified using Ni-NTA agarose from $1 \mathrm{~mL}$ iVAX reactions lasting $\sim 1$ h. Top panels show signal from probing with anti-hexa-histidine antibody (aHis) to detect the carrier protein, middle panels show signal from probing with commercial anti-FtO-PS antibody 
( $\alpha$ FtO-PS), and bottom panels show $\alpha$ His and $\alpha F t O-P S$ signals merged. Images are representative of at least three biological replicates. Dashed lines indicate samples are from the same blot with the same exposure. Molecular weight ladders are shown at the left of each image. See also Figures S3 and S4.

We next asked whether the yields of bioconjugates produced using iVAX were sufficient to enable production of relevant vaccine doses. Recent clinical data show 1-10 $\mu \mathrm{g}$ doses of bioconjugate vaccine candidates are well-tolerated and effective in stimulating the production of antibacterial IgGs (Hatz et al., 2015; Huttner et al., 2017; Riddle et al., 2016). To assess expression titers and for future experiments, we focused on $\mathrm{MBP}^{4 \times D Q N A T}$ and $\mathrm{PD}^{4 \times D Q N A T}$ because these carriers expressed in vitro with high soluble titers and without truncation products (Figure 2). We found that reactions lasting $\sim 1$ hour produced $\sim 20 \mu \mathrm{g} \mathrm{mL}^{-1}$ of glycosylated MBP $\mathrm{M}^{4 \mathrm{DQNAT}}$ and $\mathrm{PD}^{4 \times \mathrm{DQNAT}}$ as determined by ${ }^{14} \mathrm{C}$-leucine incorporation and densitometry analysis (Figure S4a). It should be noted that vaccines are currently distributed in vials containing 1-20 doses of vaccine to minimize wastage (Humphreys, 2011). Our yields suggest that multiple doses per $\mathrm{mL}$ can be synthesized in 1 hour using the iVAX platform.

To demonstrate the modularity of the iVAX approach for bioconjugate production, we sought to produce bioconjugates bearing O-PS antigens from additional pathogens including ETEC E. coli strain O78 and UPEC E. coli strain O7. E. coli $\mathrm{O} 78$ is a major cause of diarrheal disease in developing countries, especially among children, and a leading cause of traveler's diarrhea (Qadri et al., 2005), while the 07 strain is a common cause of urinary tract infections (Johnson, 1991). Like the FtO-PS, the biosynthetic pathways for EcO78-PS and EcO7-PS have been described previously and confirmed to produce O-PS antigens with the repeating units GlcNAc $_{2} \operatorname{Man}_{2}$ (Jansson et al., 1987) and Qui4NAcMan(Rha)GalGlcNAc (L'vov et al., 1984) (GlcNAc: N-acetylglucosamine; Man: mannose; Qui4NAc: 4-acetamido-4,6-dideoxy-Dglucopyranose; Rha: rhamnose; Gal: galactose), respectively. Using iVAX lysates from cells expressing CjPgIB and either the EcO78-PS and EcO7-PS pathways in reactions that were primed with $\mathrm{PD}^{4 \times D Q N A T}$ or sfGFP $217-\mathrm{DQNAT}$ plasmids, we observed carrier glycosylation only when 
both lipid-linked O-PS and CjPgIB were present in the reactions (Figure S4b, c). Our results demonstrate modular production of bioconjugates against multiple bacterial pathogens, enabled by compatibility of multiple heterologous O-PS pathways with in vitro carrier protein synthesis and glycosylation.

Endotoxin editing and freeze-drying yield iVAX reactions that are safe and portable. A key challenge inherent in using any E. coli-based system for biopharmaceutical production is the presence of lipid A, or endotoxin, which is known to contaminate protein products and can cause lethal septic shock at high levels (Russell, 2006). As a result, the amount of endotoxin in formulated biopharmaceuticals is regulated by the United States Pharmacopeia (USP), US Food and Drug Administration (FDA), and the European Medicines Agency (EMEA) (Brito and Singh, 2011). Because our iVAX reactions rely on lipid-associated components, such as CjPgIB and FtOPS, standard detoxification approaches involving the removal of lipid A (Petsch and Anspach, 2000) could compromise the activity or concentration of our glycosylation components in addition to increasing cost and processing complexities.

To address this issue, we adapted a previously reported strategy to detoxify the lipid A molecule through strain engineering (Chen et al., 2016; Needham et al., 2013). In particular, the deletion of the acyltransferase gene IpxM and the overexpression of the $F$. tularensis phosphatase LpxE in E. coli has been shown to result in the production of nearly homogenous pentaacylated, monophosphorylated lipid A with significantly reduced toxicity but retained activity as an adjuvant (Chen et al., 2016). This pentaacylated, monophosphorylated lipid A was structurally identical to the primary component of monophosphoryl lipid A (MPL) from Salmonella minnesota R595, an approved adjuvant composed of a mixture of monophosphorylated lipids (Casella and Mitchell, 2008). To generate detoxified lipid A structures in the context of iVAX, we produced lysates from a $\triangle l p x M$ derivative of CLM24 that co-expressed FtLpxE and the FtO-PS glycosylation pathway (Figure 5a). Lysates derived from this strain exhibited significantly decreased levels of toxicity 
compared to wild type CLM24 lysates expressing CjPgIB and FtO-PS (Figure 5b) as measured by human TLR4 activation in HEK-Blue hTLR4 reporter cells (Needham et al., 2013). Importantly, the structural remodeling of lipid A did not affect the activity of the membrane-bound CjPgIB and FtO-PS components in iVAX reactions (Figure S5a). By engineering the chassis strain for lysate production, we produced iVAX lysates with endotoxin levels $<1,000 \mathrm{EU} \mathrm{mL} \mathrm{m}^{-1}$, within the range of reported values for commercial protein-based vaccine products $\left(0.288-180,000 \mathrm{EU} \mathrm{mL}{ }^{-1}\right)($ Brito and Singh, 2011).

A major limitation of traditional conjugate vaccines is that they must be refrigerated $\mathrm{WHO}$, 2014), making it difficult to distribute these vaccines to remote or resource-limited settings. The ability to freeze-dry iVAX reactions for ambient temperature storage and distribution could alleviate the logistical challenges associated with refrigerated supply chains that are required for existing vaccines. To investigate this possibility, detoxified iVAX lysates were used to produce FtO-PS bioconjugates in two different ways: either by running the reaction immediately after priming with plasmid encoding the sfGFP ${ }^{217-D Q N A T}$ target protein or by running after the same reaction mixture was lyophilized and rehydrated (Figure 5c). In both cases, conjugation of FtOPS to sfGFP 217-DQNAT was observed when CjPgIB was present, with modification levels that were nearly identical (Figure 5d). We also showed that detoxified, freeze-dried iVAX reactions can be scaled to $5 \mathrm{~mL}$ for production of FtO-PS-conjugated $\mathrm{MBP}^{4 \times D Q N A T}$ and $\mathrm{PD}^{4 \times D Q N A T}$ in a manner that was reproducible from lot to lot and indistinguishable from production without freeze-drying (Figure S5b, c). The ability to lyophilize iVAX reactions and manufacture bioconjugates without specialized equipment highlights the potential for portable, on-demand vaccine production. 

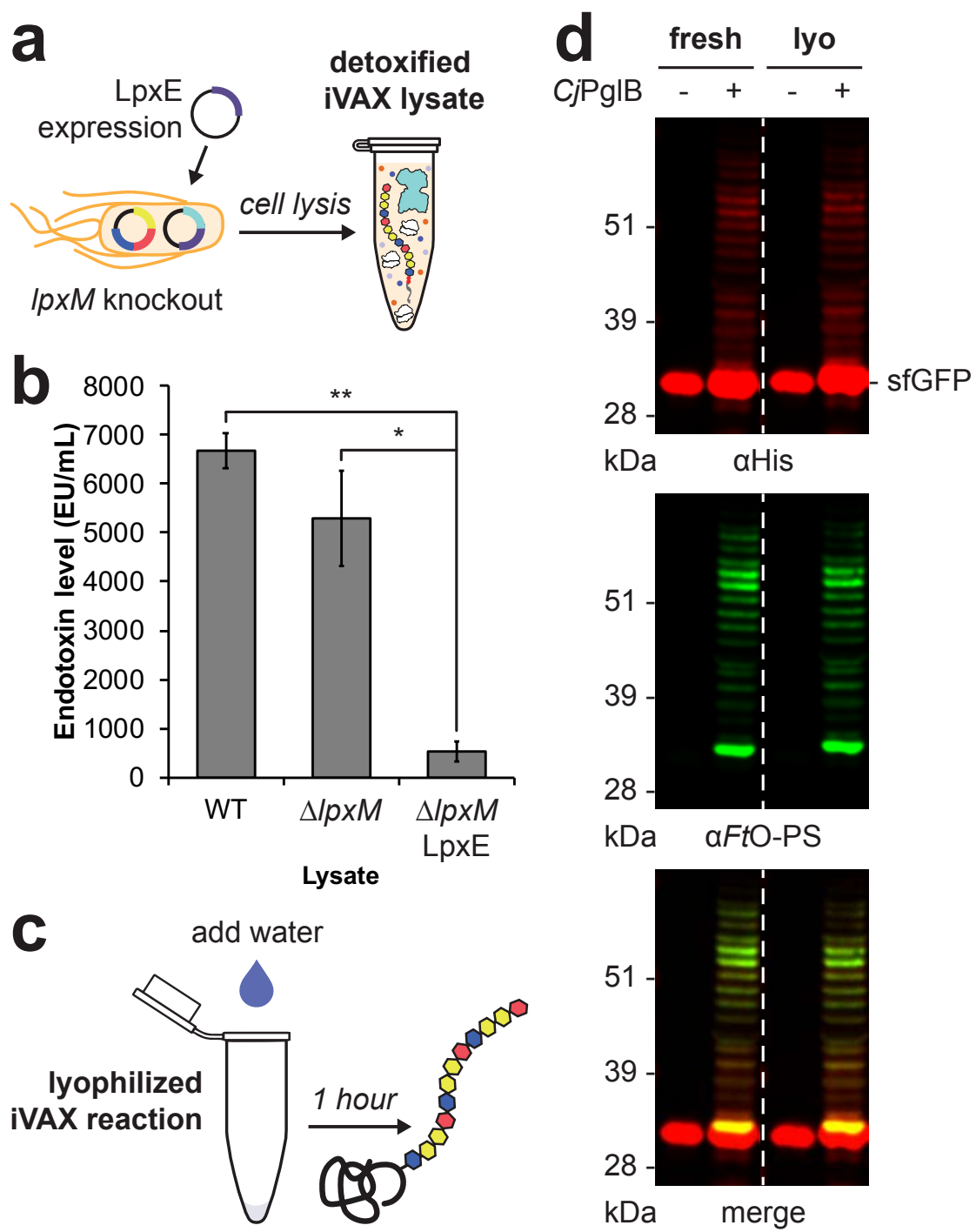

Figure 5. Detoxified, lyophilized iVAX reactions produce bioconjugates. (a) iVAX lysates were detoxified via deletion of IpxM and expression of $F$. tularensis LpxE in the source strain for lysate production. (b) The resulting lysates exhibited significantly reduced endotoxin activity. ${ }^{*} p=$ 0.019 and ${ }^{* *} p=0.003$, as determined by two-tailed $t$-test. (c) iVAX reactions producing sfGFP ${ }^{217-}$ DQNAT were run immediately or following lyophilization and rehydration. (d) Glycosylation activity was preserved following lyophilization, demonstrating the potential of iVAX reactions for portable biosynthesis of bioconjugate vaccines. Top panel shows signal from probing with anti-hexahistidine antibody ( $\mathrm{aHis}$ ) to detect the carrier protein, middle panel shows signal from probing with commercial anti-FtO-PS antibody ( $\alpha$ FtO-PS), and bottom panel shows aHis and aFtO-PS signals merged. Images are representative of at least three biological replicates. Molecular weight ladder is shown at the left of each image. See also Figure S5.

In vitro synthesized bioconjugates elicit pathogen-specific antibodies in mice. To validate the efficacy of bioconjugates produced using the iVAX platform, we next evaluated the ability of 
iVAX-derived bioconjugates to elicit anti-FtLPS antibodies in mice (Figure 6a). Importantly, we found that BALB/c mice receiving iVAX-derived FtO-PS-conjugated MBP ${ }^{4 \times D Q N A T}$ or PD ${ }^{4 \times D Q N A T}$ produced high titers of FtLPS-specific IgG antibodies, which were significantly elevated compared to the titers measured in the sera of control mice receiving PBS or aglycosylated versions of each carrier protein (Figure 6b, Figure S6). Interestingly, the IgG titers measured in sera from mice receiving glycosylated $\mathrm{MBP}^{4 \times D Q N A T}$ derived from $\mathrm{PGCT}$ were similar to the titers observed in the control groups (Figure 6b, Figure S6), in line with the markedly weaker glycosylation of this candidate relative to its iVAX-derived counterpart (Figure S3). Notably, both MBP ${ }^{4 \times D Q N A T}$ and PD ${ }^{4 \times D Q N A T}$ bioconjugates produced using iVAX elicited similar levels of IgG production and neither resulted in any observable adverse events in mice, confirming the modularity and safety of the technology for production of bioconjugate vaccine candidates.

We further characterized IgG titers by analysis of IgG1 and IgG2a subtypes and found that both iVAX-derived FtO-PS-conjugated MBP ${ }^{4 \times D Q N A T}$ and $\mathrm{PD}^{4 \times D Q N A T}$ boosted production of IgG1 antibodies by $>2$ orders of magnitude relative to all control groups as well as to glycosylated MBP $^{4 \times D Q N A T}$ derived from PGCT (Figure 6c). This analysis also revealed that both iVAX-derived bioconjugates elicited a strongly Th2-biased (lgG1 >> lgG2a) response, which is characteristic of most conjugate vaccines (Bogaert et al., 2004). Taken together, these results provide evidence that the iVAX platform supplies vaccine candidates that are capable of eliciting strong, pathogenspecific humoral immune responses and recapitulate the Th2 bias that is characteristic of licensed conjugate vaccines. 

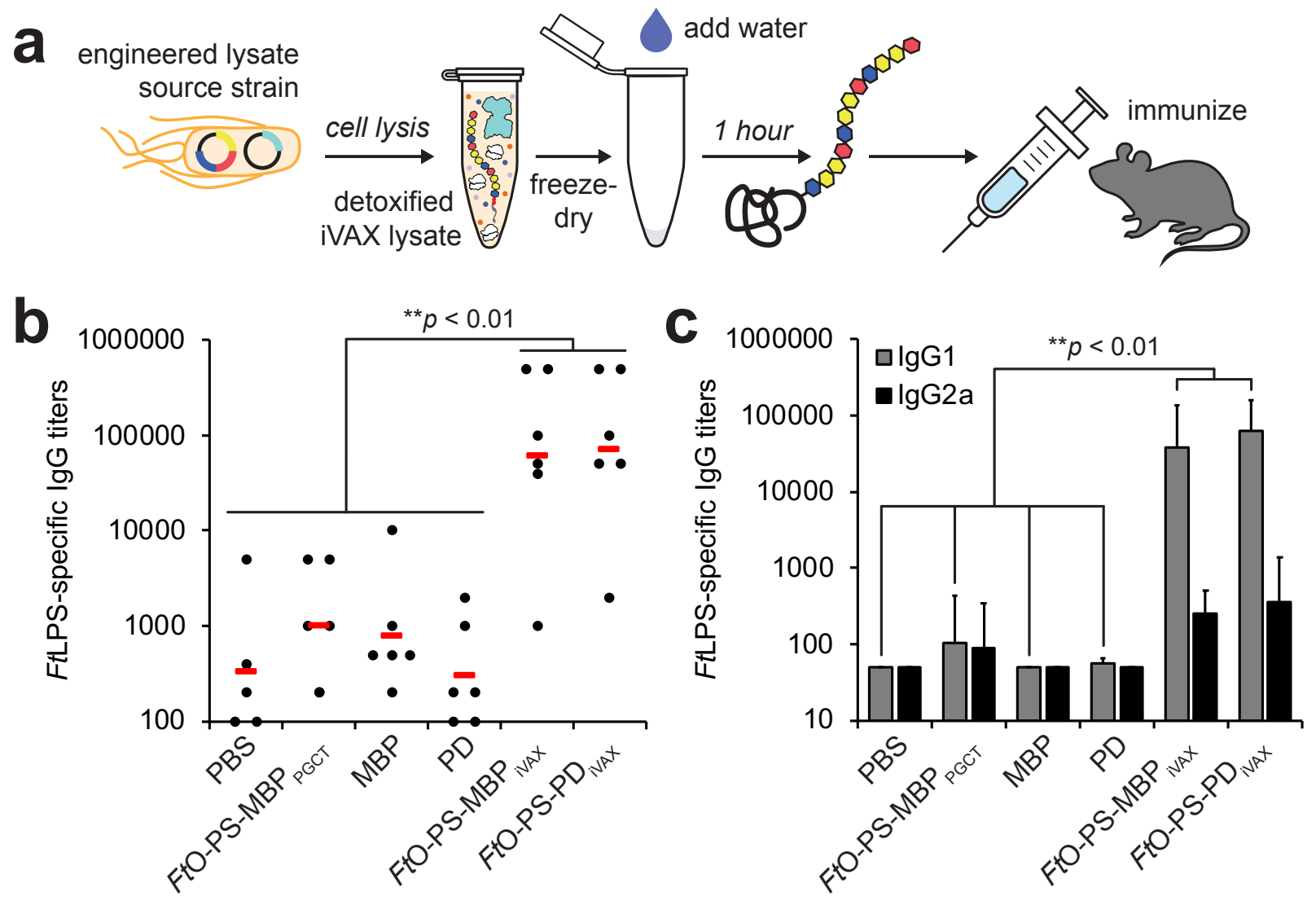

Figure 6. iVAX-derived bioconjugates elicit FtLPS-specific antibodies in mice. (a) Freezedried iVAX reactions assembled using detoxified lysates were used to synthesize anti- $F$. tularensis bioconjugates for immunization studies. (b) Six groups of BALB/c mice were immunized subcutaneously with PBS or $7.5 \mu \mathrm{g}$ of purified, cell-free synthesized aglycosylated MBP ${ }^{4 \times D Q N A T}$, FtO-PS-conjugated MBP ${ }^{4 \times D Q N A T}$, aglycosylated $\mathrm{PD}^{4 \times D Q N A T}$, or FtO-PS-conjugated PD ${ }^{4 \times D Q N A T}$. FtOPS-conjugated $\mathrm{MBP}^{4 \times D Q N A T}$ prepared in living E. coli cells using PCGT was used as a positive control. Each group was composed of six mice except for the PBS control group, which was composed of five mice. Mice were boosted on days 21 and 42 with identical doses of antigen. FtLPS-specific IgG titers were measured by ELISA in endpoint (day 70) serum of individual mice (black dots) with F. tularensis LPS immobilized as antigen. Mean titers of each group are also shown (red lines). iVAX-derived bioconjugates elicited significantly higher levels of FtLPS-specific IgG compared to all other groups ( ${ }^{* *} p<0.01$, Tukey-Kramer HSD). (c) IgG1 and IgG2a subtype titers measured by ELISA from endpoint serum revealed that iVAX-derived bioconjugates boosted production of FtO-PS-specific IgG1 compared to all other groups tested $\left({ }^{* *} p<0.01\right.$, Tukey-Kramer HSD). These results indicate that iVAX bioconjugates elicited a Th2-biased immune response typical of most conjugate vaccines. Values represent means and error bars represent standard errors of FtLPS-specific IgGs detected by ELISA. See also Figure S6.

\section{Discussion}

In this work we have established iVAX, a cell-free platform for portable, on-demand production of bioconjugate vaccines. We show that iVAX reactions can be detoxified to ensure 
the safety of bioconjugate vaccine products, freeze-dried for cold chain-independent distribution, and re-activated for high-yielding bioconjugate production by simply adding water. As a model vaccine candidate, we show that anti-F. tularensis bioconjugates derived from freeze-dried, endotoxin-edited iVAX reactions elicited pathogen-specific IgG antibodies in mice as part of a Th2-biased immune response characteristic of licensed conjugate vaccines.

The iVAX platform has several important features. First, iVAX is modular, which we have demonstrated through the interchangeability of (i) carrier proteins, including those used in licensed conjugate vaccines, and (ii) bacterial O-PS antigens from F. tularensis subsp. tularensis (type A) Schu S4, ETEC E. coli O78, and UPEC E. coli O7. To our knowledge, this represents the first demonstration of oligosaccharyltransferase-mediated O-PS conjugation to authentic FDA-approved carrier proteins, likely due to historical challenges associated with the expression of licensed carriers in living E. coli (Figueiredo et al., 1995; Haghi et al., 2011; Stefan et al., 2011). Further expansion of the O-PS pathways used in iVAX should be possible given the commonly observed clustering of polysaccharide biosynthesis genes in the genomes of pathogenic bacteria (Raetz and Whitfield, 2002). This feature could make iVAX an attractive option for rapid, de novo development of bioconjugate vaccine candidates in response to a disease outbreak or against emerging drug-resistant bacteria.

Second, iVAX reactions are inexpensive, costing $\sim \$ 12 \mathrm{~mL}^{-1}$ (Table S1) with the ability to synthesize $\sim 20 \mu \mathrm{g}$ bioconjugate $\mathrm{mL}^{-1}$ in one hour (Figure S4a). Assuming a dose size of $10 \mu \mathrm{g}$, our iVAX reactions can produce a vaccine dose for $\sim \$ 6$. For comparison, the CDC cost per dose for conjugate vaccines ranges from $\sim \$ 9.50$ for the $H$. influenzae vaccine $A c t H I B^{\circledR}$ to $\sim \$ 75$ and $\sim \$ 118$ for the meningococcal vaccine Menactra ${ }^{\circledR}$ and pneumococcal vaccine Prevnar $13^{\circledR}$, respectively (CDC, 2019).

Third, and rather interestingly, we observed that iVAX-derived bioconjugates were significantly more effective at eliciting FtLPS-specific IgGs than a bioconjugate derived from living E. coli cells using PGCT (Figure 6). One possible explanation for this increased effectiveness is 
the more extensive glycosylation that we observed for the in vitro expressed bioconjugates, with greater carbohydrate loading and decoration with higher molecular weight FtO-PS species compared to their PGCT-derived counterparts. The reduced presence of high molecular weight O-PS species observed on bioconjugates produced using PGCT could be due to competition between the O-antigen polymerase Wzy and PgIB in vivo. In contrast, our in vitro approach decouples O-PS synthesis, which occurs in vivo before lysate production, from glycosylation, which occurs in vitro as part of iVAX reactions. Our results are consistent with previous reports of PGCT-derived anti-F. tularensis bioconjugates which show that increasing the ratio of conjugated FtO-PS to protein in bioconjugates yields enhanced protection against $F$. tularensis Schu S4 in a rat inhalation model of tularemia (Marshall et al., 2018). In addition, a recent study showed that a conjugate vaccine made with a high molecular weight $(80 \mathrm{kDa})$ FtO-PS coupled to TT conferred better protection against intranasal challenge with $F$. tularensis live vaccine strain compared to a conjugate made with a low molecular weight (25 kDa) polysaccharide (Stefanetti et al., 2019). These results as well as our own point to the fact that a deeper understanding of important immunogen design features such as glycan loading and chain length could enable the production of more effective conjugate vaccines in the future.

Importantly, by enabling portable production of bioconjugate vaccines, iVAX addresses a key gap in both cell-free and decentralized biomanufacturing technologies. Production of glycosylated products has not yet been demonstrated in cell-based decentralized biomanufacturing platforms (Crowell et al., 2018; Perez-Pinera et al., 2016) and existing cell-free platforms using E. coli lysates lack the ability to synthesize glycoproteins (Boles et al., 2017; Murphy et al., 2019; Pardee et al., 2016; Salehi et al., 2016). While glycosylated human erythropoietin has been produced in a cell-free biomanufacturing platform based on freeze-dried Chinese hamster ovary cell lysates (Adiga et al., 2018), this and the vast majority of other eukaryotic cell-free and cell-based systems rely on endogenous protein glycosylation machinery. As a result, these expression platforms offer little control over the glycan structures produced or 
the underlying glycosylation reactions, and significant optimization is often required to achieve acceptable glycosylation profiles (Adiga et al., 2018). In contrast, the iVAX platform is enabled by lysates derived from $E$. coli that lack endogenous protein glycosylation pathways, allowing for bottom-up engineering of desired glycosylation activity (Jaroentomeechai et al., 2018). The ability to engineer desired glycosylation activity in iVAX uniquely enables the production of an important class of antibacterial vaccines at the point-of-care.

In summary, iVAX provides a new approach for rapid development and portable, ondemand biomanufacturing of bioconjugate vaccines. The iVAX platform alleviates cold chain requirements, which could enhance delivery of medicines to regions with limited infrastructure and minimize vaccine losses due to spoilage. In addition, the ability to rapidly produce vaccine candidates in vitro provides a unique means for rapidly responding to pathogen outbreaks and emergent threats. As a result, we believe that the iVAX platform, along with an emerging set of technologies with the ability to synthesize biomedicines on-demand (Adiga et al., 2018; Boles et al., 2017; Crowell et al., 2018; Murphy et al., 2019; Pardee et al., 2016; Perez-Pinera et al., 2016; Salehi et al., 2016), has the potential to promote increased access to complex, life-saving drugs through decentralized production.

\section{Acknowledgements}

This work was supported by Defense Threat Reduction Agency (HDTRA1-15-10052/P00001 to M.P.D. and M.C.J.), National Science Foundation (Grants \# CBET 1159581 and CBET 1264701 both to M.P.D. and MCB 1413563 to M.P.D. and M.C.J.), the David and Lucile Packard Foundation, and the Dreyfus Teacher-Scholar program. J.C.S. and T.C.S. were supported by National Science Foundation Graduate Research Fellowships. T.J. was supported by a Royal Thai Government Fellowship. R.S.D. was funded, in part, by the Northwestern University Chemistry of Life Processes Summer Scholars program. 


\section{Author Contributions}

J.C.S. designed research, performed research, analyzed data, and wrote the paper. T.J. and T.M. designed research, performed research, and analyzed data. R.S.D. and K.J.H. performed research. T.C.S. aided in research design. M.C.J. and M.P.D. directed research, analyzed data, and wrote the paper.

\section{Declaration of Interests}

M.P.D. has a financial interest in Glycobia, Inc. and Versatope, Inc. M.P.D.'s interests are reviewed and managed by Cornell University in accordance with their conflict of interest policies. All other authors declare no competing financial interests.

\section{STAR Methods}

\section{Contact for Reagent and Resource Sharing}

For further information and request of reagents please address the lead contact Michael Jewett (m-jewett@northwestern.edu).

\section{Experimental Model and Subject Details}

\section{Bacteria}

E. coli strains were grown in Luria Bertani (LB), LB-Lennox, or $2 x Y T P$ media at $30-37^{\circ} \mathrm{C}$ in shaking incubators, as specified in the Method Details section below. E. coli NEB 5-alpha (NEB) was used for plasmid cloning and purification. E. coli CLM24 or CLM24 $\Delta / p x M$ strains were used for preparing cell-free lysates. E. coli CLM24 was used as the chassis for expressing bioconjugates in vivo using PGCT. CLM24 is a glyco-optimized derivative of W3110 that carries a deletion in the 
gene encoding the WaaL ligase, facilitating the accumulation of preassembled glycans on undecaprenyl diphosphate (Feldman et al., 2005). CLM24 $\Delta / p x M$ has an endogenous acyltransferase deletion and serves as the chassis strain for production of detoxified cell-free lysates.

\section{Human cell culture}

HEK-Blue hTLR4 cells (Invivogen) were cultured in DMEM media high glucose/L-glutamine supplement with $10 \%$ fetal bovine serum, $50 \mathrm{U} \mathrm{mL}^{-1}$ penicillin, $50 \mathrm{mg} \mathrm{mL}^{-1}$ streptomycin, and 100 $\mu \mathrm{g} \mathrm{mL} \mathrm{mL}^{-1}$ Normacin ${ }^{\mathrm{TM}}$ in a humidified incubator at $37^{\circ} \mathrm{C}$ with $5 \% \mathrm{CO}_{2}$, per manufacturer's specifications.

Mice

Six-week old female BALB/c mice obtained from Harlan Sprague Dawley were randomly assigned to experimental groups. Mice were housed in groups of three to five animals and cages were changed weekly. All animals were observed daily for behavioral and physical health such as visible signs of injury and abnormal grooming habits. Mice were also observed 24 and 48 hours after each injection and no abnormal responses were reported. This study was performed at Cornell University in facilities accredited by the Association for Assessment and Accreditation of Laboratory Animal Care International. All procedures were done in accordance with Protocol 2012-0132 approved by the Cornell University Institutional Animal Care and Use Committee under relevant university, state, and federal regulations including the Animal Welfare Act, Public Health Service Policy on Humane Care and Use of Laboratory Animals, New York State Health Law Article 5, Section 504, and Cornell University Policy 1.4.

\section{Method Details}

Construction of CLM24 $\Delta / p x M$ strain 
E. coli CLM24 $\Delta / p \times M$ was generated using the Datsenko-Wanner gene knockout method (Datsenko and Wanner, 2000). Briefly, CLM24 cells were transformed with the pKD46 plasmid encoding the $\lambda$ red system. Transformants were grown to an $\mathrm{OD}_{600}$ of $0.5-0.7$ in $25 \mathrm{~mL}$ LB-Lennox media (10 $\mathrm{g} \mathrm{L}^{-1}$ tryptone, $5 \mathrm{~g} \mathrm{~L}^{-1}$ yeast extract and $5 \mathrm{~g} \mathrm{~L}^{-1} \mathrm{NaCl}$ ) with $50 \mu \mathrm{g} \mathrm{mL}^{-1}$ carbenicillin at $30^{\circ} \mathrm{C}$, harvested and washed three times with $25 \mathrm{~mL}$ ice-cold $10 \%$ glycerol to make them electrocompetent, and resuspended in a final volume of $100 \mu \mathrm{L} 10 \%$ glycerol. In parallel, a IpxM knockout cassette was generated by PCR amplifying the kanamycin resistance cassette from pKD4 with forward and reverse primers with homology to IpxM. Electrocompetent cells were transformed with $400 \mathrm{ng}$ of the IpxM knockout cassette and plated on LB agar with $30 \mu \mathrm{mL}^{-1}$ kanamycin for selection of resistant colonies. Plates were grown at $37^{\circ} \mathrm{C}$ to cure cells of the pKD46 plasmid. Colonies that grew on kanamycin were confirmed to have acquired the knockout cassette via colony PCR and DNA sequencing. These confirmed colonies were then transformed with pCP20 to remove the kanamycin resistance gene via Flp-FRT recombination. Transformants were plated on LB agar with $50 \mu \mathrm{g} \mathrm{mL}^{-1}$ carbenicillin. Following selection, colonies were grown in liquid culture at $42^{\circ} \mathrm{C}$ to cure cells of the pCP20 plasmid. Colonies were confirmed to have lost both IpxM and the knockout cassette via colony PCR and DNA sequencing and confirmed to have lost both kanamycin and carbenicillin resistance via replica plating on LB agar plates with $50 \mu \mathrm{g}$ $\mathrm{mL}^{-1}$ carbenicillin or kanamycin. All primers used for construction and validation of this strain are listed in Table S2.

All plasmids used in the study are listed in Table S3. Plasmids pJL1-MBP ${ }^{4 \times D Q N A T}$, pJL1$\mathrm{PD}^{4 \times D Q N A T}$, pJL1-PorA ${ }^{4 \times D Q N A T}$, pJL1-TTc ${ }^{4 \times D Q N A T}$, pJL1-TTlight ${ }^{4 \times D Q N A T}, p J L 1-C R M 197^{4 \times D Q N A T}$, and pJL1-TT $4 \times$ DQNAT were generated via PCR amplification and subsequent Gibson Assembly of a codon optimized gene construct purchased from IDT with a C-terminal 4xDQNAT-6xHis tag (Fisher et al., 2011) between the Ndel and Sall restriction sites in the pJL1 vector. Plasmid pJL1EPA ${ }^{\text {DNNNS-DQNRT }}$ was constructed using the same approach, but without the addition of a C-terminal 4xDQNAT-6xHis tag. Plasmids pTrc99s-ssDsbA-MBP ${ }^{4 \times D Q N A T}$, pTrc99s-ssDsbA-PD ${ }^{4 \times D Q N A T}$, 
pTrc99s-ssDsbA-PorA ${ }^{4 \times D Q N A T}$, pTrc99s-ssDsbA-TTc ${ }^{4 \times D Q N A T}$, pTrc99s-ssDsbA-TTlight $^{4 \times D Q N A T}$, and pTrc99s-ssDsbA-EPA ${ }^{\text {DNNNS-DQNRT }}$ were created via PCR amplification of each carrier protein gene and insertion into the pTrc99s vector between the $\mathrm{Ncol}$ and HindIII restriction sites via Gibson Assembly. Plasmid pSF-CjPgIB-LpxE was constructed using a similar approach, but via insertion of the IpxE gene from pE (Needham et al., 2013) between the Ndel and Nsil restriction sites in the pSF vector. Inserts were amplified via PCR using Phusion ${ }^{\circledR}$ High-Fidelity DNA polymerase (NEB) with forward and reverse primers designed using the NEBuilder $®$ Assembly Tool (nebuilder.neb.com) and purchased from IDT. The pJL1 vector (Addgene 69496) was digested using restriction enzymes $\mathrm{Ndel}$ and Sall-HF® (NEB). The pSF vector was digested using restriction enzymes Ndel and Notl (NEB). PCR products were gel extracted using an EZNA Gel Extraction Kit (Omega Bio-Tek), mixed with Gibson assembly reagents and incubated at $50^{\circ} \mathrm{C}$ for 1 hour. Plasmid DNA from the Gibson assembly reactions were transformed into E. coli NEB 5alpha cells and circularized constructs were selected using kanamycin at $50 \mu \mathrm{g} \mathrm{ml}^{-1}$ (Sigma). Sequence-verified clones were purified using an EZNA Plasmid Midi Kit (Omega Bio-Tek) for use in CFPS and iVAX reactions.

\section{Cell-free lysate preparation}

E. coli CLM24 source strains were grown in 2xYTP media (10 g/L yeast extract, $16 \mathrm{~g} / \mathrm{L}$ tryptone, $5 \mathrm{~g} / \mathrm{L} \mathrm{NaCl}, 7 \mathrm{~g} / \mathrm{L} \mathrm{K}_{2} \mathrm{HPO}_{4}, 3 \mathrm{~g} / \mathrm{L} \mathrm{KH}_{2} \mathrm{PO}_{4}, \mathrm{pH} 7.2$ ) in shake flasks (1 L scale) or a Sartorius Stedim BIOSTAT Cplus bioreactor ( $10 \mathrm{~L} \mathrm{scale}$ ) at $37^{\circ} \mathrm{C}$. Protein synthesis yields and glycosylation activity were reproducible across different batches of lysate at both small and large scale. To generate CjPgIB-enriched lysate, CLM24 cells carrying plasmid pSF-CjPgIB (Ollis et al., 2015) was used as the source strain. To generate FtO-PS-enriched lysates, CLM24 carrying plasmid pGAB2 (Cuccui et al., 2013) was used as the source strain. To generate one-pot lysates containing both CjPgIB and FtO-PS, EcO78-PS, or EcO7-PS, CLM24 carrying pSF-CjPgIB and one of the following bacterial O-PS biosynthetic pathway plasmids was used as the source strain: pGAB2 
(FtO-PS), pMW07-O78 (EcO78-PS), and pJHCV32 (EcO7-PS). CjPgIB expression was induced at an $\mathrm{OD}_{600}$ of $0.8-1.0$ with $0.02 \%(\mathrm{w} / \mathrm{v}) \mathrm{L}$-arabinose and cultures were moved to $30^{\circ} \mathrm{C}$. Cells were grown to a final $\mathrm{OD}_{600}$ of $\sim 3.0$, at which point cells were pelleted by centrifugation at $5,000 \mathrm{xg}$ for $15 \mathrm{~min}$ at $4^{\circ} \mathrm{C}$. Cell pellets were then washed three times with cold S30 buffer (10 mM Tris-acetate $\mathrm{pH} 8.2,14 \mathrm{mM}$ magnesium acetate, $60 \mathrm{mM}$ potassium acetate) and pelleted at $5000 \mathrm{xg}$ for $10 \mathrm{~min}$ at $4^{\circ} \mathrm{C}$. After the final wash, cells were pelleted at $7000 \mathrm{xg}$ for $10 \mathrm{~min}$ at $4^{\circ} \mathrm{C}$, weighed, flash frozen in liquid nitrogen, and stored at $-80^{\circ} \mathrm{C}$. To make cell lysate, cell pellets were resuspended to homogeneity in $1 \mathrm{~mL}$ of $\mathrm{S} 30$ buffer per $1 \mathrm{~g}$ of wet cell mass. Cells were disrupted via a single passage through an Avestin EmulsiFlex-B15 (1 L scale) or EmulsiFlex-C3 (10 L scale) highpressure homogenizer at $20,000-25,000$ psi. The lysate was then centrifuged twice at $30,000 \times g$ for $30 \mathrm{~min}$ to remove cell debris. Supernatant was transferred to clean microcentrifuge tubes and incubated at $37^{\circ} \mathrm{C}$ with shaking at $250 \mathrm{rpm}$ for $60 \mathrm{~min}$. Following centrifugation $(15,000 \mathrm{xg})$ for 15 min at $4^{\circ} \mathrm{C}$, supernatant was collected, aliquoted, flash-frozen in liquid nitrogen, and stored at $80^{\circ} \mathrm{C}$. S30 lysate was active for about 3 freeze-thaw cycles and contained $\sim 40 \mathrm{~g} / \mathrm{L}$ total protein as measured by Bradford assay.

\section{Cell-free protein synthesis}

CFPS reactions were carried out in $1.5 \mathrm{~mL}$ microcentrifuge tubes ( $15 \mu \mathrm{L}$ scale), $15 \mathrm{~mL}$ conical tubes ( $1 \mathrm{~mL}$ scale), or $50 \mathrm{~mL}$ conical tubes ( $5 \mathrm{~mL}$ scale) with a modified PANOx-SP system (Jewett and Swartz, 2004). The CFPS reaction mixture consists of the following components: 1.2 mM ATP; $0.85 \mathrm{mM}$ each of GTP, UTP, and CTP; $34.0 \mu \mathrm{g} \mathrm{mL} \mathrm{L}^{-1} \mathrm{~L}-5$-formyl-5, 6, 7, 8tetrahydrofolic acid (folinic acid); $170.0 \mu \mathrm{g} \mathrm{mL} \mathrm{m}^{-1}$ of $E$. coli tRNA mixture; $130 \mathrm{mM}$ potassium glutamate; $10 \mathrm{mM}$ ammonium glutamate; $12 \mathrm{mM}$ magnesium glutamate; $2 \mathrm{mM}$ each of 20 amino acids; $0.4 \mathrm{mM}$ nicotinamide adenine dinucleotide (NAD); $0.27 \mathrm{mM}$ coenzyme-A (CoA); $1.5 \mathrm{mM}$ spermidine; $1 \mathrm{mM}$ putrescine; $4 \mathrm{mM}$ sodium oxalate; $33 \mathrm{mM}$ phosphoenolpyruvate (PEP); $57 \mathrm{mM} \mathrm{HEPES;} 13.3 \mu \mathrm{g} \mathrm{mL} \mathrm{mL}^{-1}$ plasmid; and $27 \% \mathrm{v} / \mathrm{v}$ of cell lysate. For reaction volumes 
$\geq 1 \mathrm{~mL}$, plasmid was added at $6.67 \mu \mathrm{g} \mathrm{mL}{ }^{-1}$, as this lower plasmid concentration conserved reagents with no effect on protein synthesis yields or kinetics. For expression of PorA, reactions were supplemented with nanodiscs at $1 \mu \mathrm{g} \mathrm{mL}^{-1}$, which were prepared as previously described (Schoborg et al., 2017) or purchased (Cube Biotech). For expression of CRM197 $4 \times$ DQNAT, CFPS was carried out at $25^{\circ} \mathrm{C}$ for 20 hours, unless otherwise noted. For all other carrier proteins, CFPS was run at $30^{\circ} \mathrm{C}$ for 20 hours, unless otherwise noted.

For expression of $\mathrm{TT}^{4 \times \mathrm{DQNAT}}$, which contains intermolecular disulfide bonds, CFPS was carried out under oxidizing conditions. For oxidizing conditions, lysate was pre-conditioned with $750 \mu \mathrm{M}$ iodoacetamide at room temperature for $30 \mathrm{~min}$ to covalently bind free sulfhydryls (-SH) and the reaction mix was supplemented with $200 \mathrm{mM}$ glutathione at a $4: 1$ ratio of oxidized and reduced forms and $10 \mu \mathrm{M}$ recombinant E. coli DsbC (Knapp et al., 2007).

In vitro bioconjugate vaccine expression (iVAX)

For in vitro expression and glycosylation of carrier proteins in crude lysates, a two-phase scheme was implemented. In the first phase, CFPS was carried out for $15 \mathrm{~min}$ at $25-30^{\circ} \mathrm{C}$ as described above. In the second phase, protein glycosylation was initiated by the addition of $\mathrm{MnCl}_{2}$ and $\mathrm{DDM}$ at a final concentration of $25 \mathrm{mM}$ and $0.1 \%(\mathrm{w} / \mathrm{v})$, respectively, and allowed to proceed at $30^{\circ} \mathrm{C}$ for a total reaction time of 1 hour. Protein synthesis yields and glycosylation activity were reproducible across biological replicates of iVAX reactions at both small and large scale. Reactions were then centrifuged at 20,000xg for $10 \mathrm{~min}$ to remove insoluble or aggregated protein products and the supernatant was analyzed by SDS-PAGE and Western blotting.

Purification of aglycosylated and glycosylated carriers from iVAX reactions was carried out using Ni-NTA agarose (Qiagen) according to manufacturer's protocols. Briefly, $0.5 \mathrm{~mL}$ Ni-NTA agarose per $1 \mathrm{~mL}$ cell-free reaction mixture was equilibrated in Buffer 1 (300 mM NaCl $50 \mathrm{mM}$ $\mathrm{NaH}_{2} \mathrm{PO}_{4}$ ) with $10 \mathrm{mM}$ imidazole. Soluble fractions from iVAX reactions were loaded on Ni-NTA agarose and incubated at $4^{\circ} \mathrm{C}$ for $2-4$ hours to bind $6 x$ His-tagged protein. Following incubation, 
the cell-free reaction/agarose mixture was loaded onto a polypropylene column (BioRad) and washed twice with 6 column volumes of Buffer 1 with $20 \mathrm{mM}$ imidazole. Protein was eluted in 4 fractions, each with $0.3 \mathrm{~mL}$ Buffer 1 with $300 \mathrm{mM}$ imidazole per $\mathrm{mL}$ of cell-free reaction mixture. All buffers were used and stored at $4^{\circ} \mathrm{C}$. Protein was stored at a final concentration of $1-2 \mathrm{mg} \mathrm{mL}^{-}$ ${ }^{1}$ in sterile 1 XPBS (137 mM NaCl, $2.7 \mathrm{mM} \mathrm{KCl}, 10 \mathrm{mM} \mathrm{Na}_{2} \mathrm{HPO}_{4}, 1.8 \mathrm{mM} \mathrm{KH}_{2} \mathrm{PO}_{4}, \mathrm{pH} 7.4$ ) at $4^{\circ} \mathrm{C}$.

Expression of bioconjugates in vivo using protein glycan coupling technology (PGCT)

Plasmids encoding bioconjugate carrier protein genes preceded by the DsbA leader sequence for translocation to the periplasm were transformed into CLM24 cells carrying pGAB2 and pSFCjPgIB. CLM24 carrying only pGAB2 was used as a negative control. Transformed cells were grown in $5 \mathrm{~mL}$ LB media ( $10 \mathrm{~g} \mathrm{~L}^{-1}$ yeast extract, $5 \mathrm{~g} \mathrm{~L}^{-1}$ tryptone, $5 \mathrm{~g} \mathrm{~L}^{-1} \mathrm{NaCl}$ ) overnight at $37^{\circ} \mathrm{C}$. The next day, cells were subcultured into $100 \mathrm{~mL}$ LB and allowed to grow at $37^{\circ} \mathrm{C}$ for 6 hours after which the culture was supplemented with $0.2 \%$ arabinose and $0.5 \mathrm{mM}$ IPTG to induce expression of CjPgIB and the bioconjugate carrier protein, respectively. Protein expression was then carried out for 16 hours at $30^{\circ} \mathrm{C}$, at which point cells were harvested. Cell pellets were resuspended in 1 $\mathrm{mL}$ sterile PBS (137 mM NaCl, $2.7 \mathrm{mM} \mathrm{KCl}, 10 \mathrm{mM} \mathrm{Na}_{2} \mathrm{HPO}_{4}, 1.8 \mathrm{mM} \mathrm{KH}_{2} \mathrm{PO}_{4}, \mathrm{pH}$ 7.4) and lysed using a Q125 Sonicator (Qsonica, Newtown, CT) at 40\% amplitude in cycles of $10 \mathrm{sec}$ on/10 sec off for a total of $5 \mathrm{~min}$. Soluble fractions were isolated following centrifugation at 15,000 rpm for $30 \mathrm{~min}$ at $4^{\circ} \mathrm{C}$. Protein was purified from soluble fractions using Ni-NTA spin columns (Qiagen), following the manufacturer's protocol.

\section{Western blot analysis}

Samples were run on 4-12\% Bis-Tris SDS-PAGE gels (Invitrogen). Following electrophoretic separation, proteins were transferred from gels onto Immobilon-P polyvinylidene difluoride (PVDF) membranes $(0.45 \mu \mathrm{m})$ according to the manufacturer's protocol. Membranes were washed with PBS (80 g L $\left.{ }^{-1} \mathrm{NaCl}, 0.2 \mathrm{~g} \mathrm{~L}^{-1} \mathrm{KCl}, 1.44 \mathrm{~g} \mathrm{~L}^{-1} \mathrm{Na}_{2} \mathrm{HPO}_{4}, 0.24 \mathrm{~g} \mathrm{~L}^{-1} \mathrm{KH}_{2} \mathrm{PO}_{4}, \mathrm{pH} 7.4\right)$ 
followed by incubation for 1 hour in Odyssey® Blocking Buffer (LI-COR). After blocking, membranes were washed 6 times with PBST $\left(80 \mathrm{~g} \mathrm{~L}^{-1} \mathrm{NaCl}, 0.2 \mathrm{~g} \mathrm{~L}^{-1} \mathrm{KCl}, 1.44 \mathrm{~g} \mathrm{~L}^{-1} \mathrm{Na}_{2} \mathrm{HPO}_{4}\right.$, $0.24 \mathrm{~g} \mathrm{~L}^{-1} \mathrm{KH}_{2} \mathrm{PO}_{4}, 1 \mathrm{~mL} \mathrm{~L}-1$ Tween-20, $\mathrm{pH}$ 7.4) with a 5 min incubation between each wash. For iVAX samples, membranes were probed with both an anti-6xHis tag antibody and an anti-O-PS antibody or antisera specific to the $\mathrm{O}$ antigen of interest, if commercially available. Probing of membranes was performed for at least 1 hour with shaking at room temperature, after which membranes were washed with PBST in the same manner as described above and probed with fluorescently labeled secondary antibodies. Membranes were imaged using an Odyssey® Fc imaging system (LI-COR). CRM197 and TT production were compared to commercial DT and TT standards (Sigma) and orthogonally detected by an identical SDS-PAGE procedure followed by Western blot analysis with a polyclonal antibody that recognizes diphtheria or tetanus toxin, respectively. All antibodies and dilutions used are listed in Table S4.

\section{TLR4 activation assay}

HEK-Blue hTLR4 cells (Invivogen) were maintained in DMEM media, high glucose/L-glutamine supplement with $10 \%$ fetal bovine serum, $50 \mathrm{U} \mathrm{mL}^{-1}$ penicillin, $50 \mathrm{mg} \mathrm{mL}^{-1}$ streptomycin, and 100 $\mu \mathrm{g} \mathrm{mL}{ }^{-1}$ Normacin ${ }^{\mathrm{TM}}$ at $37^{\circ} \mathrm{C}$ in a humidified incubator containing $5 \% \mathrm{CO}_{2}$. After reaching $\sim 50-$ $80 \%$ confluency, cells were plated into 96 -well plates at a density of $1.4 \times 10^{5}$ cells per $\mathrm{mL}$ in HEK-Blue detection media (Invivogen). Antigens were added at the following concentrations: 100 $\mathrm{ng} \mu \mathrm{L}^{-1}$ purified protein; and $100 \mathrm{ng} \mu \mathrm{L}^{-1}$ total protein in lysate. Purified E. coli O55:B5 LPS (SigmaAldrich) and detoxified E. coli O55:B5 (Sigma-Aldrich) were added at $1.0 \mathrm{ng} \mathrm{mL}^{-1}$ and served as positive and negative controls, respectively. Plates were incubated at $37^{\circ} \mathrm{C}, 5 \% \mathrm{CO}_{2}$ for $10-16 \mathrm{~h}$ before measuring absorbance at $620 \mathrm{~nm}$. Statistical significance was determined using paired $t$ tests.

\section{Mouse immunization}


Six groups of six-week old female BALB/c mice (Harlan Sprague Dawley) were injected subcutaneously with $100 \mu \mathrm{L} \mathrm{PBS}(\mathrm{pH} 7.4)$ alone or containing purified aglycosylated MBP, FtOPS-conjugated MBP, aglycosylated PD, or FtO-PS-conjugated PD, as previously described (Chen et al., 2010). Groups were composed of six mice except for the PBS control group, which was composed of five mice. The amount of antigen in each preparation was normalized to $7.5 \mu \mathrm{g}$ to ensure that an equivalent amount of aglycosylated protein or bioconjugate was administered in each case. Purified protein groups formulated in PBS were mixed with an equal volume of incomplete Freund's Adjuvant (Sigma-Aldrich) before injection. Prior to immunization, material for each group $(5 \mu \mathrm{L})$ was streaked on LB agar plates and grown overnight at $37^{\circ} \mathrm{C}$ to confirm sterility and endotoxin activity was measured by TLR4 activation assay. Each group of mice was boosted with an identical dosage of antigen 21 days and 42 days after the initial immunization. Blood was obtained on day $-1,21,35,49$, and 63 via submandibular collection and at study termination on day 70 via cardiac puncture. Mice were observed 24 and 48 hours after each injection for changes in behavior and physical health and no abnormal responses were reported. This study and all procedures were done in accordance with Protocol 2012-0132 approved by the Cornell University Institutional Animal Care and Use Committee.

\section{Enzyme-linked immunosorbent assay}

F. tularensis LPS-specific antibodies elicited by immunized mice were measured via indirect ELISA using a modification of a previously described protocol (Chen et al., 2010). Briefly, sera were isolated from the collected blood draws after centrifugation at 5000xg for $10 \mathrm{~min}$ and stored at $-20^{\circ} \mathrm{C}$; 96-well plates (Maxisorp; Nunc Nalgene) were coated with $F$. tularensis LPS (BEI resources) at a concentration of $5 \mu \mathrm{gL}^{-1}$ in PBS and incubated overnight at $4^{\circ} \mathrm{C}$. The next day, plates were washed three times with PBST (PBS, 0.05\% Tween-20, 0.3\% BSA) and blocked overnight at $4^{\circ} \mathrm{C}$ with $5 \%$ nonfat dry milk (Carnation) in PBS. Samples were serially diluted by a factor of two in triplicate between $1: 100$ and $1: 12,800,000$ in blocking buffer and added to the 
plate for 2 hours at $37^{\circ} \mathrm{C}$. Plates were washed three times with PBST and incubated for 1 hour at $37^{\circ} \mathrm{C}$ in the presence of one of the following HRP-conjugated antibodies (all from Abcam and used at 1:25,000 dilution): goat anti-mouse IgG, anti-mouse IgG1, and anti-mouse IgG2a. After three additional washes with PBST, 3,3'-5,5'-tetramethylbenzidine substrate (1-Step Ultra TMBELISA; Thermo-Fisher) was added, and the plate was incubated at room temperature in the dark for $30 \mathrm{~min}$. The reaction was halted with $2 \mathrm{M} \mathrm{H}_{2} \mathrm{SO}_{4}$, and absorbance was quantified via microplate spectrophotometer (Tecan) at a wavelength of $450 \mathrm{~nm}$. Serum antibody titers were determined by measuring the lowest dilution that resulted in signal 3 SDs above no serum background controls. Statistical significance was determined in RStudio 1.1.463 using one-way ANOVA and the Tukey-Kramer post hoc honest significant difference test.

\section{Quantification and Statistical Analysis}

Quantification of cell-free protein synthesis yields

To quantify the amount of protein synthesized in iVAX reactions, two approaches were used. Fluorescence units of sfGFP were converted to concentrations using a previously reported standard curve (Hong et al., 2014). Yields of all other proteins were assessed via the addition of $10 \mu \mathrm{M} \mathrm{L-}{ }^{14} \mathrm{C}$-leucine $\left(11.1 \mathrm{GBq} \mathrm{mmol}^{-1}\right.$, PerkinElmer $)$ to the CFPS mixture to yield trichloroacetic acid-precipitable radioactivity that was measured using a liquid scintillation counter as described previously (Kim and Swartz, 2001).

\section{Statistical analysis}

Statistical parameters including the definitions and values of $n, p$-values, standard deviations, and standard errors are reported in the Figures and corresponding Figure Legends. Analytical techniques are described in the corresponding Method Details section.

\section{Data and Software Availability}


All plasmid constructs used in this study including complete DNA sequences are deposited on Addgene (constructs 128389-128404).

\section{Supplemental Information Titles and Legends}

Figure S1. In vitro synthesis of licensed conjugate vaccine carrier proteins is possible over a range of temperatures and can be readily optimized, Related to Figure 2. (a) With the exception of CRM197, all carriers expressed with similar soluble yields at $25^{\circ} \mathrm{C}, 30^{\circ} \mathrm{C}$, and $37^{\circ} \mathrm{C}$, as measured by ${ }^{14} \mathrm{C}$-leucine incorporation. Values represent means and error bars represent standard deviations of biological replicates $(n=3)$. (b) Soluble expression of PorA was improved through the addition of lipid nanodiscs to the reaction. (c) Expression of full-length TT was enhanced by (i) performing in vitro protein synthesis in oxidizing conditions to improve assembly of the disulfide-bonded heavy and light chains into full-length TT and (ii) allowing reactions to run for only $2 \mathrm{~h}$ to minimize protease degradation. (d) CRM197 and (e) TT produced in CFPS reactions are detected with $\alpha$-DT and $\alpha$-TT antibodies, respectively, and are comparable in size to commercially available purified DT and TT protein standards (50 ng standard loaded). Images are representative of at least three biological replicates. Dashed line indicates samples are from the same blot with the same exposure. Molecular weight ladders are shown at the left of each image.

Figure S2. Glycosylation in iVAX reactions occurs in $1 \mathrm{~h}$ over a range of temperatures, Related to Figure 3. Kinetics of FtO-PS glycosylation at $30^{\circ} \mathrm{C}$ (left), $37^{\circ} \mathrm{C}, 25^{\circ} \mathrm{C}$, and room temperature $\left(\sim 21^{\circ} \mathrm{C}\right)$ (right) are comparable and show that protein synthesis and glycosylation occur in the first hour of the iVAX reaction. These results demonstrate that the iVAX platform can synthesize bioconjugates over a range of permissible temperatures. Top panels show signal from probing with anti-hexa-histidine antibody ( $\alpha \mathrm{His}$ ) to detect the carrier protein, middle panels show signal from probing with commercial anti-FtO-PS antibody ( $\alpha$ FtO-PS), and bottom panels show $\alpha \mathrm{His}$ and $\alpha F t O-P S$ signals merged. Images are representative of at least three biological replicates. Molecular weight ladders are shown at the left of each image.

Figure S3. Production of bioconjugates against $F$. tularensis using PGCT in living $E$. coli, Related to Figure 4. (a) Bioconjugates were produced via PGCT in CLM24 cells expressing CjPgIB, the biosynthetic pathway for FtO-PS, and a panel of immunostimulatory carriers including those used in licensed vaccines. (b) We observed low expression of PorA, a membrane protein, as well as reduced glycan loading and conjugation of high molecular weight FtO-PS species in all carriers compared to iVAX-derived samples. Top panels show signal from probing with anti-hexahistidine antibody ( $\alpha \mathrm{His}$ ) to detect the carrier protein, middle panels show signal from probing with commercial anti-FtO-PS antibody ( $\alpha$ FtO-PS), and bottom panels show $\alpha$ His and $\alpha F t O-P S$ signals merged. Images are representative of at least three biological replicates. Molecular weight ladders are shown at the left of each image.

Figure S4. The iVAX platform is modular and can be used to synthesize clinically relevant yields of diverse bioconjugates, Related to Figure 4. (a) Protein synthesis and glycosylation with FtO-PS were measured in iVAX reactions producing MBP ${ }^{4 \times D Q N A T}$ and $P D^{4 \times D Q N A T}$. After $\sim 1 \mathrm{~h}$, reactions produced $\sim 40 \mu \mathrm{g} \mathrm{mL}{ }^{-1}$ protein, as measured via ${ }^{14} \mathrm{C}$-leucine incorporation, of which $\sim 20 \mu \mathrm{g} \mathrm{mL}^{-1}$ was glycosylated with FtO-PS, as determined by densitometry. Values represent means and error bars represent standard errors of biological replicates $(n=2)$. To demonstrate 
modularity, iVAX lysates were prepared from cells expressing CjPgIB and biosynthetic pathways for either (b) the E. coli O78 antigen or (c) the E. coli O7 antigen and used to synthesize PD ${ }^{4 \times D N A T}$ (left) or sfGFP $217-D Q N A T$ (right) bioconjugates. The structure and composition of the repeating monomer unit for each antigen is shown. Both polysaccharide antigens are compositionally and, in the case of the $\mathrm{O} 7$ antigen, structurally distinct compared to the $F$. tularensis $\mathrm{O}$ antigen. Blots show signal from probing with anti-hexa-histidine antibody (aHis) to detect the carrier protein. If a commercial anti-O-PS serum or antibody was available, it was used to confirm the identity of the conjugated $\mathrm{O}$ antigen ( $\alpha-E c \mathrm{O} 78$ blots, panel $\mathbf{b}$ ). Asterisk denotes bands resulting from nonspecific serum antibody binding. Images are representative of at least three biological replicates. Dashed lines indicate samples are from the same blot with the same exposure. Molecular weight ladders are shown at the left of each image.

Figure S5. Detoxified iVAX lysates synthesize bioconjugates and both lysate production and freeze-dried reactions scale reproducibly, Related to Figure 5. (a) iVAX lysates containing CjPgIB and FtO-PS were prepared from wild-type CLM24, CLM24 $\Delta / p x M$, or CLM24 $\Delta / p \times M$ cells expressing FtLpxE. Nearly identical sfGFP ${ }^{217-D Q N A T}$ glycosylation was observed for each of the lysates derived from the engineered strains. (b) To generate material for immunizations, fermentations to produce endotoxin-edited iVAX lysates were scaled from $0.5 \mathrm{~L}$ to $10 \mathrm{~L}$. We observed similar levels of sfGFP ${ }^{217-D Q N A T}$ glycosylation for lysates derived from $0.5 \mathrm{~L}$ and $10 \mathrm{~L}$ cultures, and across different batches of lysate produced from $10 \mathrm{~L}$ fermentations. (c) For immunizations, we prepared two lots of FtO-PS-conjugated MBP ${ }^{4 \times D Q N A T}$ and PD ${ }^{4 \times D Q N A T}$ from $5 \mathrm{~mL}$ freeze-dried iVAX reactions. We observed similar levels of purified protein $(\sim 200 \mu \mathrm{g})$ and FtO-PS modification ( $>50 \%$, measured by densitometry) across both carriers and lots of material. Top panels show signal from probing with anti-hexa-histidine antibody (aHis) to detect the carrier protein, middle panels show signal from probing with commercial anti-FtO-PS antibody ( $\alpha$ FtO-PS), and bottom panels show $\alpha$ His and $\alpha$ FtO-PS signals merged. Images are representative of at least three biological replicates. Molecular weight ladders are shown at left.

Figure S6. FtLPS-specific antibody titers in vaccinated mice over time, Related to Figure 6. Six groups of BALB/c mice were immunized subcutaneously with PBS or $7.5 \mu \mathrm{g}$ of purified, cell-free synthesized aglycosylated MBP ${ }^{4 \times D Q N A T}$, FtO-PS-conjugated MBP ${ }^{4 \times D Q N A T}$, aglycosylated $\mathrm{PD}^{4 \times D Q N A T}$, or FtO-PS-conjugated $\mathrm{PD}^{4 \times D Q N A T}$. FtO-PS-conjugated MBP ${ }^{4 \times D Q N A T}$ prepared in living $E$. coli cells using PCGT was used as a positive control. Each group was composed of six mice except for the PBS control group, which was composed of five mice. Mice were boosted on days 21 and 42 with identical doses of antigen. FtLPS-specific IgG titers were measured by ELISA in serum collected on day $-1,35,49,63$, and 70 following initial immunization. iVAX-derived bioconjugates elicited significantly higher levels of FtLPS-specific IgG compared to compared to the PBS control group in serum collected on day 35,49 , and 70 of the study ${ }^{* *} p<0.01$, TukeyKramer HSD). Values represent means and error bars represent standard errors of FtLPS-specific IgGs detected by ELISA.

Table S1. Cost analysis for iVAX reactions, Related to STAR Methods. The total cost to assemble iVAX reactions is calculated below. A $1 \mathrm{~mL}$ iVAX reaction produces two $10 \mu \mathrm{g}$ vaccine doses and can be assembled for $\$ 11.75$. In the table, amino acid cost accounts for $2 \mathrm{mM}$ each of the 20 canonical amino acids purchased individually from Sigma. Lysate cost is based on a single employee making $50 \mathrm{~mL}$ lysate from a $10 \mathrm{~L}$ fermentation, assuming 30 lysate batches per year and a 5-year equipment lifetime. Component source is also included in the table if it is available to purchase directly from a supplier. Homemade components cannot be purchased directly and must be prepared according to procedures described in the Methods section. 
Table S2. Primers used to generate CLM24 $\triangle$ IpxM, Related to STAR Methods. Primers used to construct and verify the CLM24 $\Delta / p x M$ strain are listed below. KO primers were used for amplification of the kanamycin resistance cassette from pKD4 with homology to IpxM. Seq primers were used for colony PCRs and sequencing confirmation of knockout strains.

Table S3. Plasmids used in this study, Related to STAR Methods.

Table S4. Antibodies and antisera used in this study, Related to STAR Methods.

\section{References}

Adiga, R., Al-adhami, M., Andar, A., Borhani, S., Brown, S., Burgenson, D., Cooper, M.A., Deldari, S., Frey, D.D., Ge, X., et al. (2018). Point-of-care production of therapeutic proteins of good-manufacturing-practice quality. Nat Biomed Eng.

Ashok, A., Brison, M., and LeTallec, Y. (2017). Improving cold chain systems: Challenges and solutions. Vaccine 35, 2217-2223.

Bayburt, T.H., and Sligar, S.G. (2010). Membrane protein assembly into Nanodiscs. FEBS Lett $584,1721-1727$.

Bhushan, R., Anthony, B.F., and Frasch, C.E. (1998). Estimation of group B streptococcus type III polysaccharide-specific antibody concentrations in human sera is antigen dependent. Infect Immun 66, 5848-5853.

Bogaert, D., Hermans, P.W., Adrian, P.V., Rumke, H.C., and de Groot, R. (2004). Pneumococcal vaccines: an update on current strategies. Vaccine 22, 2209-2220.

Boles, K.S., Kannan, K., Gill, J., Felderman, M., Gouvis, H., Hubby, B., Kamrud, K.I., Venter, J.C., and Gibson, D.G. (2017). Digital-to-biological converter for on-demand production of biologics. Nat Biotechnol 35, 672-675.

Brito, L.A., and Singh, M. (2011). Acceptable levels of endotoxin in vaccine formulations during preclinical research. J Pharm Sci 100, 34-37.

Carlson, E.D., Gan, R., Hodgman, C.E., and Jewett, M.C. (2012). Cell-free protein synthesis: Applications come of age. Biotechnol Adv 30, 1185-1194.

Casella, C.R., and Mitchell, T.C. (2008). Putting endotoxin to work for us: monophosphoryl lipid A as a safe and effective vaccine adjuvant. Cell Mol Life Sci 65, 3231-3240.

CDC (2019). CDC Vaccine Price List.

Chen, D.J., Osterrieder, N., Metzger, S.M., Buckles, E., Doody, A.M., DeLisa, M.P., and Putnam, D. (2010). Delivery of foreign antigens by engineered outer membrane vesicle vaccines. Proc Natl Acad Sci U S A 107, 3099-3104.

Chen, L., Valentine, J.L., Huang, C.-J., Endicott, C.E., Moeller, T.D., Rasmussen, J.A., Fletcher, J.R., Boll, J.M., Rosenthal, J.A., Dobruchowska, J., et al. (2016). Outer membrane vesicles displaying engineered glycotopes elicit protective antibodies. Proc Natl Acad Sci U S A. 
Chen, M.M., Glover, K.J., and Imperiali, B. (2007). From peptide to protein: comparative analysis of the substrate specificity of $\mathrm{N}$-linked glycosylation in C. jejuni. Biochemistry 46, 55795585.

Crowell, L.E., Lu, A.E., Love, K.R., Stockdale, A., Timmick, S.M., Wu, D., Wang, Y.A., Doherty, W., Bonnyman, A., Vecchiarello, N., et al. (2018). On-demand manufacturing of clinical-quality biopharmaceuticals. Nat Biotechnol.

Cuccui, J., Thomas, R.M., Moule, M.G., D'Elia, R.V., Laws, T.R., Mills, D.C., Williamson, D., Atkins, T.P., Prior, J.L., and Wren, B.W. (2013). Exploitation of bacterial N-linked glycosylation to develop a novel recombinant glycoconjugate vaccine against Francisella tularensis. Open Biol 3, 130002.

Datsenko, K.A., and Wanner, B.L. (2000). One-step inactivation of chromosomal genes in Escherichia coli K-12 using PCR products. Proc Natl Acad Sci U S A 97, 6640-6645.

Feldman, M.F., Wacker, M., Hernandez, M., Hitchen, P.G., Marolda, C.L., Kowarik, M., Morris, H.R., Dell, A., Valvano, M.A., and Aebi, M. (2005). Engineering N-linked protein glycosylation with diverse $\mathrm{O}$ antigen lipopolysaccharide structures in Escherichia coli. Proc Natl Acad Sci U S A 102, 3016-3021.

Fernandez, S., Palmer, D.R., Simmons, M., Sun, P., Bisbing, J., McClain, S., Mani, S., Burgess, T., Gunther, V., and Sun, W. (2007). Potential role for Toll-like receptor 4 in mediating Escherichia coli maltose-binding protein activation of dendritic cells. Infect Immun 75, 13591363.

Figueiredo, D., Turcotte, C., Frankel, G., Li, Y., Dolly, O., Wilkin, G., Marriott, D., Fairweather, N., and Dougan, G. (1995). Characterization of recombinant tetanus toxin derivatives suitable for vaccine development. Infect Immun 63, 3218-3221.

Fisher, A.C., Haitjema, C.H., Guarino, C., Celik, E., Endicott, C.E., Reading, C.A., Merritt, J.H., Ptak, A.C., Zhang, S., and DeLisa, M.P. (2011). Production of secretory and extracellular Nlinked glycoproteins in Escherichia coli. Appl Environ Microbiol 77, 871-881.

Frasch, C.E. (2009). Preparation of bacterial polysaccharide-protein conjugates: analytical and manufacturing challenges. Vaccine 27, 6468-6470.

Fulop, M., Mastroeni, P., Green, M., and Titball, R.W. (2001). Role of antibody to lipopolysaccharide in protection against low- and high-virulence strains of Francisella tularensis. Vaccine 19, 4465-4472.

Garcia-Quintanilla, F., Iwashkiw, J.A., Price, N.L., Stratilo, C., and Feldman, M.F. (2014). Production of a recombinant vaccine candidate against Burkholderia pseudomallei exploiting the bacterial N-glycosylation machinery. Front Microbiol 5, 381.

Haghi, F., Peerayeh, S.N., Siadat, S.D., and Montajabiniat, M. (2011). Cloning, expression and purification of outer membrane protein PorA of Neisseria meningitidis serogroup B. J Infect Dev Ctries 5, 856-862.

Hatz, C.F., Bally, B., Rohrer, S., Steffen, R., Kramme, S., Siegrist, C.A., Wacker, M., Alaimo, C., and Fonck, V.G. (2015). Safety and immunogenicity of a candidate bioconjugate vaccine 
against Shigella dysenteriae type 1 administered to healthy adults: A single blind, partially randomized Phase I study. Vaccine 33, 4594-4601.

Hong, S.H., Ntai, I., Haimovich, A.D., Kelleher, N.L., Isaacs, F.J., and Jewett, M.C. (2014). Cellfree protein synthesis from a release factor 1 deficient Escherichia coli activates efficient and multiple site-specific nonstandard amino acid incorporation. ACS Synth Biol 3, 398-409.

Humphreys, G. (2011). Vaccination: rattling the supply chain (Bulletin of the World Health Organization: World Health Organization).

Huttner, A., Hatz, C., van den Dobbelsteen, G., Abbanat, D., Hornacek, A., Frolich, R., Dreyer, A.M., Martin, P., Davies, T., Fae, K., et al. (2017). Safety, immunogenicity, and preliminary clinical efficacy of a vaccine against extraintestinal pathogenic Escherichia coli in women with a history of recurrent urinary tract infection: a randomised, single-blind, placebo-controlled phase $1 \mathrm{~b}$ trial. Lancet Infect Dis.

Ihssen, J., Kowarik, M., Dilettoso, S., Tanner, C., Wacker, M., and Thony-Meyer, L. (2010). Production of glycoprotein vaccines in Escherichia coli. Microb Cell Fact 9, 61.

Jansson, P.E., Lindberg, B., Widmalm, G., and Leontein, K. (1987). Structural studies of the Escherichia coli O78 O-antigen polysaccharide. Carbohydr Res 165, 87-92.

Jaroentomeechai, T., Stark, J.C., Natarajan, A., Glasscock, C.J., Yates, L.E., Hsu, K.J., Mrksich, M., Jewett, M.C., and DeLisa, M.P. (2018). Single-pot glycoprotein biosynthesis using a cell-free transcription-translation system enriched with glycosylation machinery. Nat Commun 9, 2686.

Jewett, M.C., and Swartz, J.R. (2004). Mimicking the Escherichia coli cytoplasmic environment activates long-lived and efficient cell-free protein synthesis. Biotechnol Bioeng 86, 19-26.

Jin, C., Gibani, M.M., Moore, M., Juel, H.B., Jones, E., Meiring, J., Harris, V., Gardner, J., Nebykova, A., Kerridge, S.A., et al. (2017). Efficacy and immunogenicity of a Vi-tetanus toxoid conjugate vaccine in the prevention of typhoid fever using a controlled human infection model of Salmonella Typhi: a randomised controlled, phase 2b trial. Lancet 390, 2472-2480.

Johnson, J.R. (1991). Virulence factors in Escherichia coli urinary tract infection. Clin Microbiol $\operatorname{Rev} 4,80-128$.

Kim, D.M., and Swartz, J.R. (2001). Regeneration of adenosine triphosphate from glycolytic intermediates for cell-free protein synthesis. Biotechnol Bioeng 74, 309-316.

Knapp, K.G., Goerke, A.R., and Swartz, J.R. (2007). Cell-free synthesis of proteins that require disulfide bonds using glucose as an energy source. Biotechnol Bioeng 97, 901-908.

Kowarik, M., Young, N.M., Numao, S., Schulz, B.L., Hug, I., Callewaert, N., Mills, D.C., Watson, D.C., Hernandez, M., Kelly, J.F., et al. (2006). Definition of the bacterial N-glycosylation site consensus sequence. EMBO J 25, 1957-1966.

L'vov, V.L., Shashkov, A.S., Dmitriev, B.A., Kochetkov, N.K., Jann, B., and Jann, K. (1984). Structural studies of the O-specific side chain of the lipopolysaccharide from Escherichia coli O:7. Carbohydr Res 126, 249-259. 
Lu, Z., Madico, G., Roche, M.I., Wang, Q., Hui, J.H., Perkins, H.M., Zaia, J., Costello, C.E., and Sharon, J. (2012). Protective B-cell epitopes of Francisella tularensis O-polysaccharide in a mouse model of respiratory tularaemia. Immunology 136, 352-360.

Lydon, P., Zipursky, S., Tevi-Benissan, C., Djingarey, M.H., Gbedonou, P., Youssouf, B.O., and Zaffran, M. (2014). Economic benefits of keeping vaccines at ambient temperature during mass vaccination: the case of meningitis A vaccine in Chad. Bull World Health Organ 92, 86-92.

Ma, Z., Zhang, H., Shang, W., Zhu, F., Han, W., Zhao, X., Han, D., Wang, P.G., and Chen, M. (2014). Glycoconjugate vaccine containing Escherichia coli O157:H7 O-antigen linked with maltose-binding protein elicits humoral and cellular responses. PloS One 9, e105215.

Marshall, L.E., Nelson, M., Davies, C.H., Whelan, A.O., Jenner, D.C., Moule, M.G., Denman, C., Cuccui, J., Atkins, T.P., Wren, B.W., et al. (2018). An O-antigen glycoconjugate vaccine produced using protein glycan coupling technology is protective in an inhalational rat model of tularemia. J Immunol Res 2018, 8087916.

Murphy, T.W., Sheng, J., Naler, L.B., Feng, X., and Lu, C. (2019). On-chip manufacturing of synthetic proteins for point-of-care therapeutics. Microsyst Nanoeng 5, 13.

Needham, B.D., Carroll, S.M., Giles, D.K., Georgiou, G., Whiteley, M., and Trent, M.S. (2013). Modulating the innate immune response by combinatorial engineering of endotoxin. Proc Natl Acad Sci U S A 110, 1464-1469.

Novak, R.T., Kambou, J.L., Diomande, F.V., Tarbangdo, T.F., Ouedraogo-Traore, R., Sangare, L., Lingani, C., Martin, S.W., Hatcher, C., Mayer, L.W., et al. (2012). Serogroup A meningococcal conjugate vaccination in Burkina Faso: analysis of national surveillance data. Lancet Infect Dis 12, 757-764.

Ollis, A.A., Chai, Y., Natarajan, A., Perregaux, E., Jaroentomeechai, T., Guarino, C., Smith, J., Zhang, S., and DeLisa, M.P. (2015). Substitute sweeteners: Diverse bacterial oligosaccharyltransferases with unique N-glycosylation site preferences. Sci Rep 5, 15237.

Oyston, P.C., Sjostedt, A., and Titball, R.W. (2004). Tularaemia: bioterrorism defence renews interest in Francisella tularensis. Nat Rev Microbiol 2, 967-978.

Pardee, K., Slomovic, S., Nguyen, Peter Q., Lee, Jeong W., Donghia, N., Burrill, D., Ferrante, T., McSorley, Fern R., Furuta, Y., Vernet, A., et al. (2016). Portable, on-demand biomolecular manufacturing. Cell 167, 248-259.e212.

Perez, J.G., Stark, J.C., and Jewett, M.C. (2016). Cell-free synthetic biology: Engineering beyond the cell. Cold Spring Harb Perspect Biol.

Perez-Pinera, P., Han, N., Cleto, S., Cao, J., Purcell, O., Shah, K.A., Lee, K., Ram, R., and Lu, T.K. (2016). Synthetic biology and microbioreactor platforms for programmable production of biologics at the point-of-care. Nat Commun 7, 12211.

Petsch, D., and Anspach, F.B. (2000). Endotoxin removal from protein solutions. J Biotechnol 76, 97-119. 
Poehling, K.A., Talbot, T.R., Griffin, M.R., Craig, A.S., Whitney, C.G., Zell, E., Lexau, C.A., Thomas, A.R., Harrison, L.H., Reingold, A.L., et al. (2006). Invasive pneumococcal disease among infants before and after introduction of pneumococcal conjugate vaccine. JAMA 295, 1668-1674.

Prior, J.L., Prior, R.G., Hitchen, P.G., Diaper, H., Griffin, K.F., Morris, H.R., Dell, A., and Titball, R.W. (2003). Characterization of the $O$ antigen gene cluster and structural analysis of the $O$ antigen of Francisella tularensis subsp. tularensis. J Med Microbiol 52, 845-851.

Qadri, F., Svennerholm, A.M., Faruque, A.S., and Sack, R.B. (2005). Enterotoxigenic Escherichia coli in developing countries: epidemiology, microbiology, clinical features, treatment, and prevention. Clin Microbiol Rev 18, 465-483.

Raetz, C.R., and Whitfield, C. (2002). Lipopolysaccharide endotoxins. Annu Rev Biochem 71, 635-700.

Riddle, M.S., Kaminski, R.W., Di Paolo, C., Porter, C.K., Gutierrez, R.L., Clarkson, K.A., Weerts, H.E., Duplessis, C., Castellano, A., Alaimo, C., et al. (2016). Safety and immunogenicity of a candidate bioconjugate vaccine against Shigella flexneri $2 a$ administered to healthy adults: a single blind, randomized phase I study. Clin Vaccine Immunol.

Roush, S.W., Mclntyre, L., and Baldy, L.M. (2008). Manual for the surveillance of vaccinepreventable diseases. Atlanta: Centers for Disease Control and Prevention, 4.

Russell, J.A. (2006). Management of sepsis. N Engl J Med 355, 1699-1713.

Salehi, A.S., Smith, M.T., Bennett, A.M., Williams, J.B., Pitt, W.G., and Bundy, B.C. (2016). Cellfree protein synthesis of a cytotoxic cancer therapeutic: Onconase production and a just-addwater cell-free system. Biotechnol J 11, 274-281.

Schoborg, J.A., Hershewe, J.M., Stark, J.C., Kightlinger, W., Kath, J.E., Jaroentomeechai, T., Natarajan, A., DeLisa, M.P., and Jewett, M.C. (2017). A cell-free platform for rapid synthesis and testing of active oligosaccharyltransferases. Biotechnol Bioeng.

Stefan, A., Conti, M., Rubboli, D., Ravagli, L., Presta, E., and Hochkoeppler, A. (2011). Overexpression and purification of the recombinant diphtheria toxin variant CRM197 in Escherichia coli. J Biotechnol 156, 245-252.

Stefanetti, G., Okan, N., Fink, A., Gardner, E., and Kasper, D.L. (2019). Glycoconjugate vaccine using a genetically modified $\mathrm{O}$ antigen induces protective antibodies to Francisella tularensis. Proc Natl Acad Sci U S A 116, 7062-7070.

The Review on Antimicrobial Resistance, C.b.J.O.N. (2014). Antimicrobial Resistance: Tackling a crisis for the health and wealth of nations.

Trotter, C.L., McVernon, J., Ramsay, M.E., Whitney, C.G., Mulholland, E.K., Goldblatt, D., Hombach, J., and Kieny, M.P. (2008). Optimising the use of conjugate vaccines to prevent disease caused by Haemophilus influenzae type b, Neisseria meningitidis and Streptococcus pneumoniae. Vaccine 26, 4434-4445. 
Wacker, M., Wang, L., Kowarik, M., Dowd, M., Lipowsky, G., Faridmoayer, A., Shields, K., Park, S., Alaimo, C., Kelley, K.A., et al. (2014). Prevention of Staphylococcus aureus infections by glycoprotein vaccines synthesized in Escherichia coli. J Infect Dis 209, 1551-1561.

Wahl, B., O'Brien, K.L., Greenbaum, A., Majumder, A., Liu, L., Chu, Y., Luksic, I., Nair, H., McAllister, D.A., Campbell, H., et al. (2018). Burden of Streptococcus pneumoniae and Haemophilus influenzae type $b$ disease in children in the era of conjugate vaccines: global, regional, and national estimates for 2000-15. Lancet Glob Health 6, e744-e757.

Weintraub, A. (2003). Immunology of bacterial polysaccharide antigens. Carbohydr Res 338, 2539-2547.

Wetter, M., Kowarik, M., Steffen, M., Carranza, P., Corradin, G., and Wacker, M. (2013). Engineering, conjugation, and immunogenicity assessment of Escherichia coli $0121 \mathrm{O}$ antigen for its potential use as a typhoid vaccine component. Glycoconj J 30, 511-522.

WHO (2014). Temperature Sensitivity of Vaccines. 